ARTICLE

\title{
Evidence that the TRPV1 S1-S4 membrane domain contributes to thermosensing
}

\author{
Minjoo Kim (1) 1,2,5, Nicholas J. Sisco 1,2,5, Jacob K. Hilton ${ }^{1,2}$, Camila M. Montano ${ }^{2}$, Manuel A. Castro (1) 1, \\ Brian R. Cherry ${ }^{3}$, Marcia Levitus ${ }^{1,4} \&$ Wade D. Van Horn (iD ${ }^{1,2}{ }^{\infty}$
}

Sensing and responding to temperature is crucial in biology. The TRPV1 ion channel is a wellstudied heat-sensing receptor that is also activated by vanilloid compounds, including capsaicin. Despite significant interest, the molecular underpinnings of thermosensing have remained elusive. The TRPV1 S1-S4 membrane domain couples chemical ligand binding to the pore domain during channel gating. Here we show that the S1-S4 domain also significantly contributes to thermosensing and couples to heat-activated gating. Evaluation of the isolated human TRPV1 S1-S4 domain by solution NMR, far-UV CD, and intrinsic fluorescence shows that this domain undergoes a non-denaturing temperature-dependent transition with a high thermosensitivity. Further NMR characterization of the temperature-dependent conformational changes suggests the contribution of the S1-S4 domain to thermosensing shares features with known coupling mechanisms between this domain with ligand and $\mathrm{pH}$ activation. Taken together, this study shows that the TRPV1 S1-S4 domain contributes to TRPV1 temperature-dependent activation.

\footnotetext{
${ }^{1}$ School of Molecular Sciences, Arizona State University, 551 E. University Drive, Tempe, AZ 85287, USA. ${ }^{2}$ The Biodesign Institute Virginia G. Piper Center for Personalized Diagnostics, Arizona State University, Tempe, AZ 85287, USA. ${ }^{3}$ The Magnetic Resonance Research Center, Arizona State University, Tempe, AZ 85287, USA. ${ }^{4}$ The Biodesign Institute Center for Single Molecule Biophysics, Arizona State University, Tempe, AZ 85287, USA.

${ }^{5}$ These authors contributed equally: Minjoo Kim, Nicholas J. Sisco. ${ }^{凶}$ email: wade.van.horn@asu.edu
} 
T ransient receptor potential (TRP) ion channels are a family of membrane proteins that play diverse physiological roles $^{1,2}$. TRPV1, from the vanilloid subfamily, is responsive to various chemical and physical stimuli, including vanilloid ligands, elevated temperature, protons, endogenous lipids, and small modulatory membrane proteins ${ }^{3-6}$. While TRPV1 is expressed in neuronal and non-neuronal tissues, its role in neuronal tissues has garnered significant interest ${ }^{7}$. For example, in group $\mathrm{C}$ unmyelinated nerve tissue of the peripheral nervous system, TRPV1 is integral to nociception (pain) ${ }^{8,9}$. Consequentially, there is interest in TRPV1 therapeutic intervention for various pain indications ${ }^{8}$. Beyond nociception, an increasing number of studies provide emerging evidence that TRPV1 is involved in diverse human physiologies and pathophysiologies including: inflammatory diseases ${ }^{10-12}$, obesity ${ }^{13,14}$, diabetes ${ }^{11}$, longevity ${ }^{15}$, and cancer ${ }^{16,17}$.

Cryo-electron microscopy (cryo-EM)-based structural biology has had a significant impact on understanding the molecular architecture that underlies TRP channel function ${ }^{18}$. These structures have shown that TRP channels resemble the transmembrane topology of voltage-gated ion channels (VGICs), with two conserved transmembrane structural domains. The S1-S4 transmembrane helices (S1-S4 domain) form a four-helix bundle that is structurally related to the voltage-sensing domain (VSD) in VGICs. In TRP channels, like their evolutionary ancestors in the VGIC superfamily, the S5-S6 transmembrane helices form the pore domain (PD), which assembles into a tetrameric channel. In addition to identifying topological features, a number of TRPV1 structures have been determined in different states, which have provided significant insight into the molecular basis for TRPV1 chemical activation ${ }^{19-21}$. Canonical TRPV1 vanilloid compounds, like the pungent agonist capsaicin, bind to the S1-S4 domain which couples with the PD to open the lower and upper gates of the channel ${ }^{22}$, thereby initiating signal transduction. The cryo-EM determined vanilloid binding site ${ }^{19-21}$ is consistent with previous studies ${ }^{23-28}$, which identified residues in the S3 and S4 helices of the TRPV1 S1-S4 domain as central for capsaicin activation.

Several TRP channels are exquisitely sensitive to changes in temperature and function as molecular thermometers. The temperature-induced activation of thermosensitive TRP channels generates large changes in enthalpy $(\Delta \mathrm{H})$ and significant compensating changes in entropy $(\Delta S)$, resulting in biologically accessible changes in free energy $(\Delta G)$ between closed and open states. The thermosensitivity of biological systems is typically assessed by measuring the temperature coefficient $\left(Q_{10}\right)$, which can be defined as the ratio of equilibrium constants measured $10^{\circ} \mathrm{C}$ apart. The temperature coefficient is related to the change in enthalpy ${ }^{29}$, and thermosensitive TRP channels like TRPV1 have large $Q_{10}$ and $\Delta \mathrm{H}$ values of $\sim 40$ and $\sim 100 \mathrm{kcal} \mathrm{mol}^{-1}$, respectively ${ }^{30,31}$. The physical mechanisms that underlie temperature sensitivity are thought to arise from changes in secondary structure and hydrophobic/hydrophilic accessibility, both of which have large thermodynamic signatures ${ }^{1,29,32,33}$. Notwithstanding, the region (or regions) within TRP channels that are key to thermosensing remains controversial. As a result, the specific biochemical and structural mechanisms that give rise to TRP channel temperature sensing are also not well understood. However, important contributions have been made to investigating TRPV1 thermosensing. For example, studies of the purified TRPV1 channel clearly show that the channel is intrinsically thermosensitive ${ }^{34}$. Similarly, an isoform of murine TRPV1 that lacks the majority of the $\mathrm{N}$-terminus remains thermosensitive ${ }^{35}$, suggestive that the transmembrane region is central to thermosensing. More recently, a chimeric study that swapped the TRPV1 PD into a non-thermosensing channel was able to endow thermosensitivity in the chimeric channel ${ }^{36}$. Despite these studies, the mechanism of TRPV1 thermosensing has remained elusive.

Given the role of the TRPV1 S1-S4 domain (V1-S1S4) in ligand activation and the structural similarity to VSDs in VGICs, we hypothesize that the S1-S4 domain may contribute to the thermosensitivity of TRPV1. We adopt a distinct strategy that is not reliant on mutagenesis but instead focuses on direct temperature-dependent characterization of the hV1-S1S4, an evolutionarily conserved structural domain (Fig. 1). Here, we show that an isolated hV1-S1S4 is in a biologically relevant state, is sufficient for vanilloid ligand binding, and retains the expected secondary structure and membrane topology. Temperaturedependent studies of the hV1-S1S4 using solution nuclear magnetic resonance (NMR) spectroscopy identify a two-state transition between folded conformational states. The magnitude of the NMR-detected temperature sensitivity $(\Delta \mathrm{H})$ is supported by farUV circular dichroism (CD) and intrinsic tryptophan fluorescence spectroscopy. Quantitative comparison with whole-cell patch-clamp electrophysiology experiments in mammalian cells indicates that the hV1-S1S4 significantly contributes to TRPV1 thermosensitivity. Additionally, the temperature-dependent conformational changes were examined by NMR-based temperaturedependent distance measurements from paramagnetic relaxation enhancement (PRE), secondary structure measurements from chemical shift assignment and residual dipolar coupling, and solvent exposure from deuterium/hydrogen exchange and water-protein NOEs. To validate these outcomes, we introduced a mutation that disrupts coupling between the S1-S4 and pore domains and show that both ligand and temperature activation are abrogated in the full-length channel but retained in the isolated domain. Taken together, the data provide thermodynamic and mechanistic insight into the properties of thermosensitive TRP channels and suggest an overlap between TRPV1 ligand, proton $(\mathrm{pH})$, and temperature activation.

\section{Results}

The isolated hV1-S1S4 resides in a biologically relevant state. The transmembrane hV1-S1S4 construct includes the pre-S1 helix through the S4 helix (residues 417 to 558) (Fig. 1a). Optimization of hV1-S1S4 expression resulted in $~ 1.5 \mathrm{mg}$ of purified protein per liter of M9 media. The identity of the hV1-S1S4 was verified by SDS-PAGE, western blot, LC-MS/MS, and ultimately NMR resonance assignment (Supplementary Fig. 1). After membrane mimic screening, the hV1-S1S4 was reconstituted in LPPG (1-palmitoyl-2-hydroxy-sn-glycero-3-phospho-(1'-rac-glycerol)) lysolipid micelles, which yielded high-quality NMR spectra (Supplementary Fig. 1). We focused on single-chain membrane mimics, such as LPPG, to avoid temperature-dependent phase transitions common to lipids that could interfere with temperature-dependent thermodynamic and biophysical characterization. TROSY-based 3D and 4D NMR experiments were carried out to assign the hV1-S1S4 amide backbone. Specifically, TROSY-based HNCA, HNCOCA, HNCO, HNCACB, CBCACONH, HNCACO, ${ }^{15} \mathrm{~N}$-edited-NOESY-TROSY, 4D HNCACO, and 4D HNCOCA were used to assign $87 \%$ of backbone resonances (BMRB ID: 27029, Supplementary Figs. 2, 1). The hV1-S1S4 membrane topology was determined from secondary structure derived from backbone chemical shift data (TALOS-N) and membrane accessibility from NMR-detected deuteriumhydrogen $(\mathrm{D} / \mathrm{H})$ exchange factors (Fig. 1b, c). The resulting hV1S1S4 secondary structure in solution at elevated temperature is similar to existing rat TRPV1 cryo-EM structures. Similarly, the $\mathrm{D} / \mathrm{H}$ exchange factors show that the transmembrane helices have lower exchange factors than the solvent-accessible loop regions, indicative of the expected membrane topology for this domain. 
a
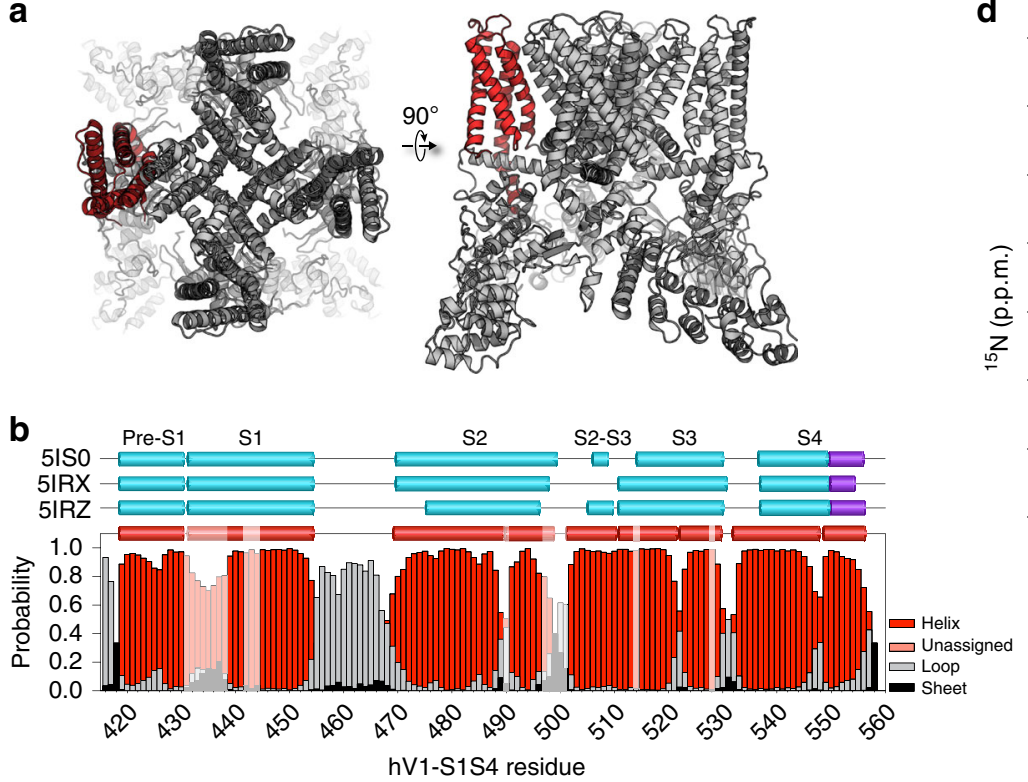

C

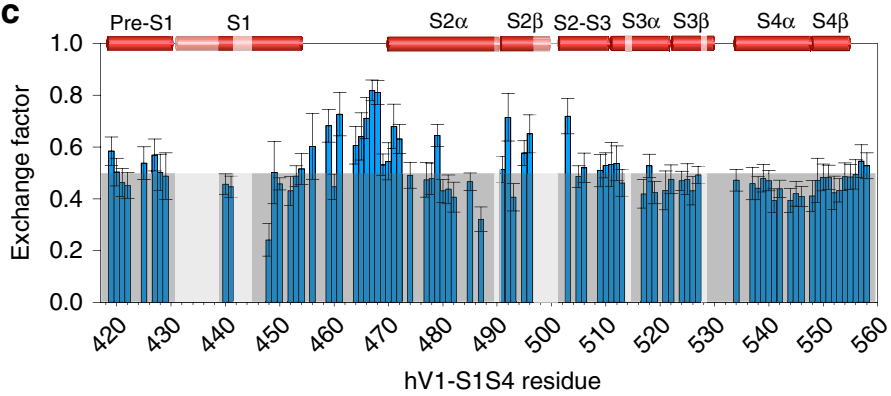

d

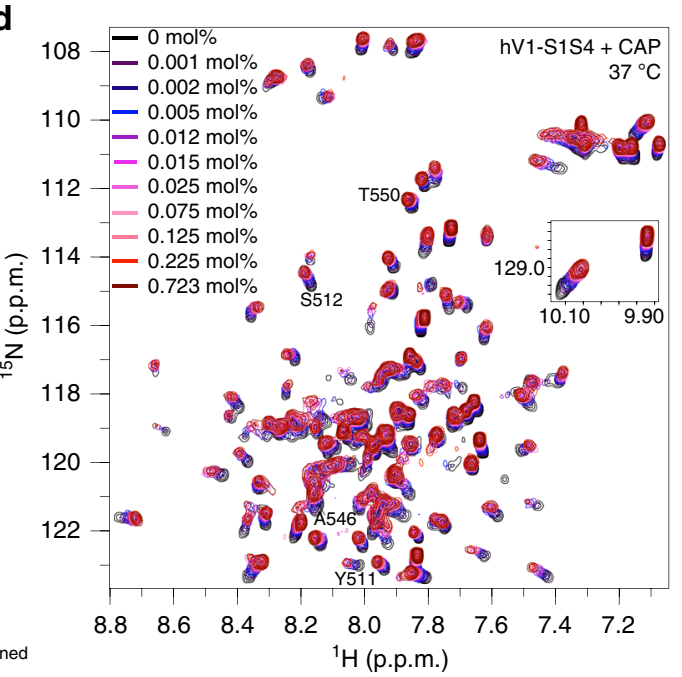

$\mathbf{e}_{1.2}$
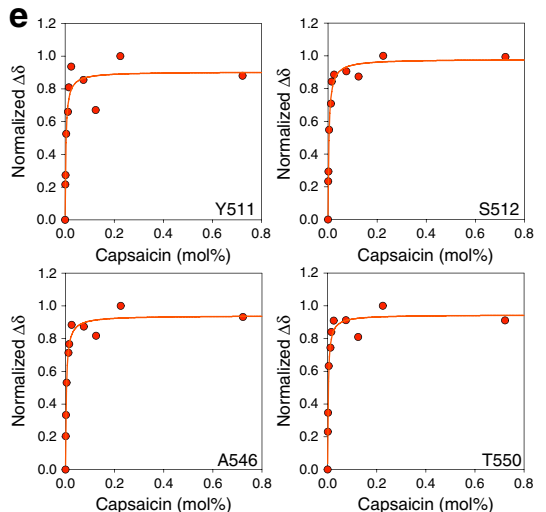

Fig. 1 The isolated human TRPV1 S1-S4 domain is folded in a biologically relevant state and binds capsaicin at elevated temperatures. a Highlighted in red is the hV1-S1S4 construct shown in the rTRPV1 cryo-EM structure (PDB ID: 3J5P) and used throughout our studies. b Cryo-EM determined secondary structure information of rTRPV1 (cyan/purple) compared with the NMR-determined secondary structure (red) from hV1-S1S4 at $45^{\circ} \mathrm{C}$ identifies the similarities and differences in secondary structure. The S4 $3_{10}$ helix is shown in purple. The unassigned region is blocked with opaque white boxes. c Deuterium/hydrogen (D/H) exchange factors show the hV1-S1S4 solvent accessibility is consistent with the anticipated membrane topology. As annotated in (b), unassigned residues are covered with opaque white boxes. Darker gray box represents the average value of exchange factors. Error bars represent per-residue fitting error. $\mathbf{d}$ Superimposed ${ }^{1} \mathrm{H}-{ }^{15} \mathrm{~N}$ HSQC spectra of the hV1-S1S4 titration with capsaicin at $37^{\circ} \mathrm{C}$. e Canonical capsaicin binding residues include $\mathrm{Y} 511, \mathrm{~S} 512, \mathrm{~T} 550$, and A546, which show saturating binding isotherms as a function of capsaicin. Source data are provided as a Source Data file.

The isolated hV1-S1S4 solubilized in LPPG retains the ability to specifically bind capsaicin at $37^{\circ} \mathrm{C}$ (Fig. 1d). Previously identified capsaicin binding residues, Y511, S512, and T550, were used as probes of capsaicin binding. These residues showed saturable chemical shift perturbation as a function of increased capsaicin concentration, which is indicative of specific binding (Fig. le). The average $K_{\mathrm{d}}$ values from the key vanilloid binding residues (Y511, S512, and T550) is $3.4 \pm 0.4 \mathrm{mmol} \%$. L547 (M547 in the rat ortholog) has also been ascribed importance in vanilloid binding and activation; however, due to resonance overlap this residue could not be analyzed. A neighboring residue, A546, which is located within $5 \AA$ of vanilloid ligand as shown a vanilloid-bound TRPV1 structure (PDB ID: 5IRX), also exhibited saturating binding with a $K_{\mathrm{d}}$ value of $3.6 \pm 0.5 \mathrm{mmol} \%$ (Fig. 1e). Global analysis of capsaicin-induced chemical shift perturbation (Supplementary Fig. 4) indicates that binding impacts regions beyond the canonical vanilloid binding site. Taken together, the isolated hV1-S1S4 in solution retains four transmembrane embedded helices and recapitulates expected features of capsaicin binding, indicating that the isolated hTRPV1-S1S4 retains a biologically relevant conformation in solution and at elevated temperatures.
Two-state thermodynamic analysis of the full-length hTRPV1. TRPV1 temperature-dependent electrophysiology data have been shown to fit well to a simple two-state thermodynamic model between open (active) and closed (resting) states ${ }^{2}$. While this is certainly an oversimplification, modeling TRPV1 temperaturedependent transitions allows for quantification of the thermosensitivity. In this two-state model, the temperature-dependent slope of the transition between conformational states reflects the change in enthalpy $(\Delta \mathrm{H})^{37}$ and is a readout of the thermosensitivity associated with the conformational change between temperatures.

To date, the majority of TRPV1 thermosensing studies have focused on rodent orthologs (Supplementary Table 1). To evaluate the thermosensitivity of the human TRPV1 ortholog, whole-cell patch-clamp electrophysiology measurements were performed using the full-length wild-type human TRPV1 in HEK293 cells. We employed two distinct methods to measure the thermosensitivity $(\Delta \mathrm{H})$ of human TRPV1 in cellular conditions. First, steady-state current values were measured at $+60 \mathrm{mV}$ as a function of increasing temperature (Supplementary Fig. 5). The normalized current values were plotted against temperature and 

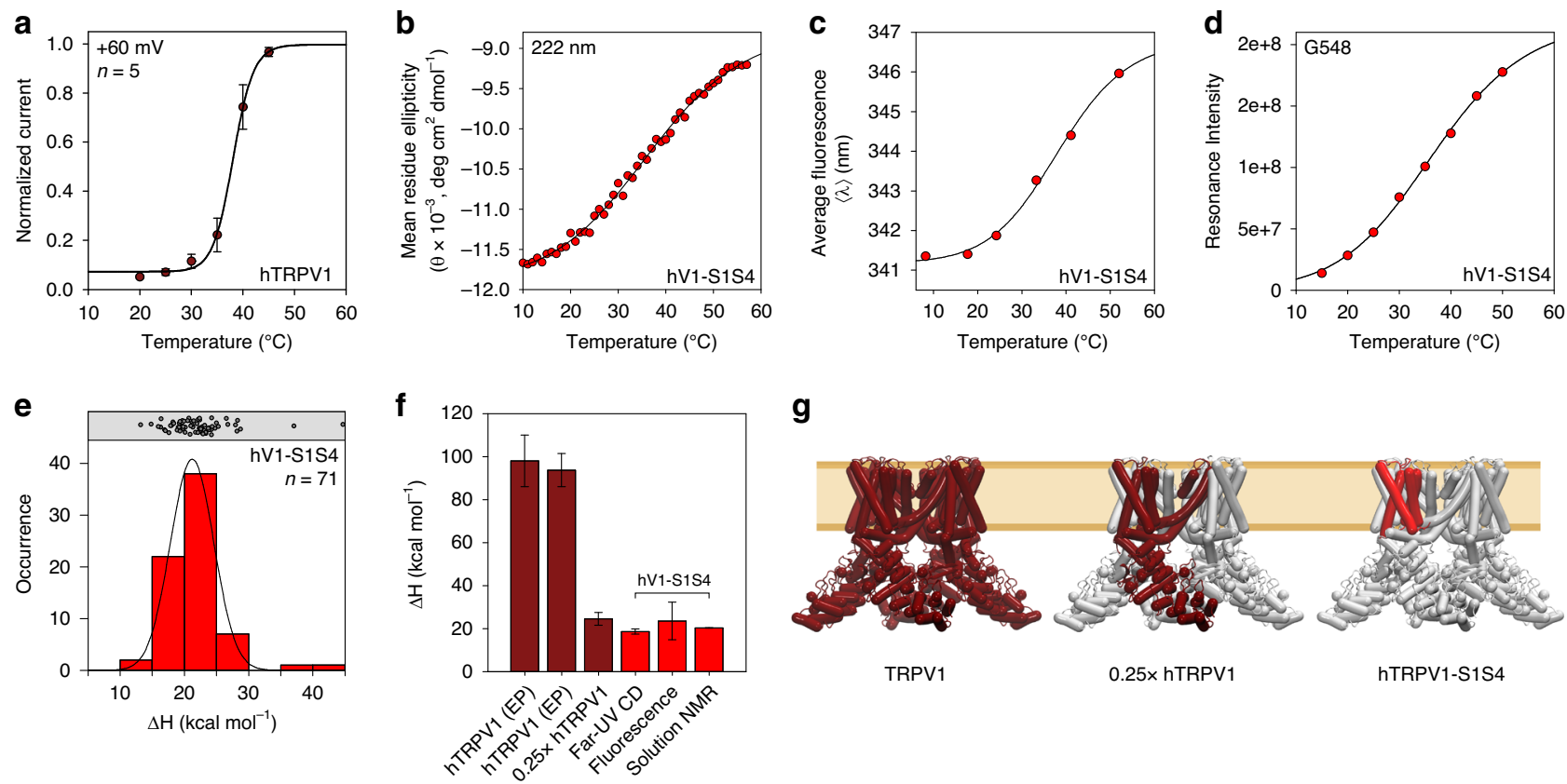

g

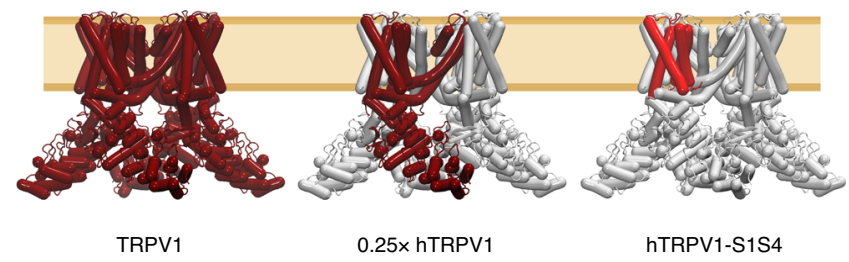

Fig. 2 Temperature-dependent two-state behavior and thermodynamic analysis of hTRPV1 and hV1-S1S4. a Whole-cell patch-clamp electrophysiology measurements from full-length human TRPV1 in HEK293 cells. b Temperature ramp of hV1-S1S4 monitored by far-UV circular dichroism shows two-state behavior. c The first moment of intrinsic tryptophan fluorescence of hV1-S1S4 as a function of temperature shows two-state transition similar to that identified by CD. d Representative NMR resonance (peak) intensity, G548 in the S4 helix, shows two-state behavior. G548 was chosen due to its similarity to the mean $\Delta \mathrm{H}$ obtained from NMR. e A histogram of 71 enthalpies from NMR resonance (peak) intensities are shown in red with individual data points shown above as black circles. These data fit to a Gaussian function (black line) provide an average value of $21.2 \pm 0.1 \mathrm{kcal}$ mol-1. f Comparison of $\Delta \mathrm{H}$ values, the first two dark red bars are from the cellular electrophysiology measurements for full-length hTRPV1 from steady-state currents (a) and temperature ramps. A calculated value for one quarter of the steady-state value is also shown. The $\triangle H$ values from CD, fluorescence, and NMR are shown in red. $\mathbf{g}$ The left panel shows the full-length TRPV1 used for electrophysiology measurements. The middle panel shows the relative size of a TRPV1 monomer. The right panel shows the hV1-S1S4 domain in red, which has similar $\Delta \mathrm{H}$ as the monomer, showing the relative significance of the hV1-S1S4 in thermosensitivity. The mid-point of the temperature dependence, $T_{50}$, for the resulting electrophysiology (a), CD (b), fluorescence (c), and NMR (d, e) are $38.1 \pm 0.3,36 \pm 1,38 \pm 2$, and $40.7 \pm 0.6^{\circ} \mathrm{C}$ respectively. Errors represent s.e.m. and source data are provided as a Source Data file.

fit to a two-state model representing closed (resting) and open (active) states resulting in a measured $\Delta \mathrm{H}$ of $98 \pm 12 \mathrm{kcal} \mathrm{mol}^{-1}$ (Fig. 2a), with a $\mathrm{T}_{50}$ of $38.1 \pm 0.3^{\circ} \mathrm{C}$. Independently, we used a second method involving temperature ramps from 20 to $50^{\circ} \mathrm{C}$ and fit the data to a pseudo-steady-state thermodynamic model to obtain hTRPV1 $\Delta \mathrm{H}$ values (see Methods), resulting in an average $\Delta \mathrm{H}$ of $94 \pm 8 \mathrm{kcal} \mathrm{mol}^{-1}$ (Supplementary Fig. 5). The $\Delta \mathrm{H}$ values of human TRPV1 measured from the steady-state current and the temperature ramp methods are consistent with those of rodent TRPV1 from previous studies, which is $\sim 90 \mathrm{kcal} \mathrm{mol}^{-1}$ (Supplementary Table 1).

Two-state thermodynamic analysis of the hV1-S1S4 domain. To investigate a potential role of the hV1-S1S4 in thermosensitivity, far-UV circular dichroism (CD) and solution NMR spectra were recorded at $20^{\circ} \mathrm{C}$ and $50^{\circ} \mathrm{C}$; temperatures that would be expected to correspond to distinct conformational states if the hV1-S1S4 were to contribute to thermosensitivity. First, the $\mathrm{CD}$ spectra at $20^{\circ} \mathrm{C}$ and $50^{\circ} \mathrm{C}$ displayed characteristic a-helical minima at 208 and $222 \mathrm{~nm}$. These spectral features were retained at both high and low temperatures, suggestive that the protein does not significantly unfold under these conditions (Supplementary Fig. 5). Additionally, the ${ }^{1} \mathrm{H}_{-}{ }^{15} \mathrm{~N}$ TROSY-HSQC NMR spectra of hV1-S1S4 at these two temperatures $\left(20\right.$ and $\left.50{ }^{\circ} \mathrm{C}\right)$ provide qualitative insight into potential temperature-dependent conformational change and the foldedness of this domain at the respective temperatures (Supplementary Fig. 5). The NMR proton resonance dispersion and resolution indicate that the hV1-S1S4 remains generally folded over this temperature range. There is also significant temperature-dependent chemical shift perturbation between the spectra at 20 and $50^{\circ} \mathrm{C}$, which is consistent with a temperature-induced conformational change. The temperaturedependent spectral changes, in both $\mathrm{CD}$ and NMR, are completely reversible and indicate that the system is suitable for thermodynamic analysis to assess thermosensitivity $(\Delta \mathrm{H})$ of the apparent conformational change (Supplementary Methods and Fig. 5).

$\mathrm{CD}$ data collected as a function of temperature have long been used to determine thermodynamic properties of proteins ${ }^{37}$. The hV1-S1S4 CD data exhibit two-state behavior monitored at 222 $\mathrm{nm}$, near the characteristic $a$-helical minimum, as a function of temperature. Fitting the data to a two-state sigmoidal model yielded a $\Delta \mathrm{H}$ of $19 \pm 1 \mathrm{kcal} \mathrm{mol}^{-1} \quad\left(T_{50}=36 \pm 1{ }^{\circ} \mathrm{C}\right)$, which reflects the temperature dependence of the hV1-S1S4 change in conformational states (Fig. 2b). The KCNQ1-VSD is structurally homologous to the hV1-S1S4; however, it is not directly activated (gated) by thermal stimulus. As a control, we measured the KCNQ1-VSD mean residue ellipticity (MRE) at $222 \mathrm{~nm}$ over the same temperature range. The hKCNQ1-VSD did not exhibit twostate behavior, but instead showed a linear temperature response over this temperature range, consistent with a general thermal expansion of the a-helical hydrogen bonds (Supplementary Fig. 6). Comparatively, the lack of measurable thermosensitivity $(\Delta \mathrm{H})$ of the KCNQ1-VSD over this temperature range suggests that the hV1-S1S4 reflects a feature of TRPV1 thermosensitivity.

The hV1-S1S4 was also subjected to temperature-dependent studies using intrinsic tryptophan fluorescence. Typically, intrinsic 
tryptophan has a large fluorescence transition dipole moment and the position and shape of the emission spectrum is particularly sensitive to the polarity of the environment. Tryptophan (Trp) emission maxima in an apolar (hydrophobic) environment is blue-shifted and varies from ca. $310-335 \mathrm{~nm}$. Whereas emission maxima in polar (hydrogen bonding) solvents for an unstructured Trp residue is red-shifted about $40 \mathrm{~nm}$ up to ca. $355 \mathrm{~nm}^{38}$. These characteristics are commonly exploited in protein folding studies, where the emission peak of Trp shifts towards the red as the fraction of unfolded protein increases. In the context of hV1-S1S4, this analysis allows insight into both the thermosensitivity of the transition and the environment of the Trp residues. The hV1-S1S4 has two endogenous Trp residues in the amphipathic pre-S1 helix and the transmembrane S4 helix, both of which are anticipated to be in a hydrophobic environment from cryo-EM structures. Indeed, the resulting fluorescence emission spectral maxima are $328 \mathrm{~nm}$ at $7.4^{\circ} \mathrm{C}$ and $332 \mathrm{~nm}$ at $52.0^{\circ} \mathrm{C}$, indicating that over the temperature range studied, the endogenous $\operatorname{Trp}$ residues generally retain their membrane embeddedness (Supplementary Fig. 6). This observation is consistent with the observations from CD (Supplementary Fig. 5) and NMR (Supplementary Fig. 6) that the hV1-S1S4 remains generally folded across this temperature range. Quantifying the observed temperature-induced spectral shifts in terms of the average emission wavelength $(\langle\lambda\rangle)$ identifies a sigmoidal twostate behavior with a $\Delta \mathrm{H}$ of $24 \pm 5 \mathrm{kcal} \mathrm{mol}^{-1}$ (Fig. 2c) and a $T_{50}$ of $38 \pm 2{ }^{\circ} \mathrm{C}$. This thermosensitivity is consistent with the observed CD-detected thermosensitivity (Fig. 2b).

A series of ${ }^{1} \mathrm{H}-{ }^{15} \mathrm{~N}$ TROSY-HSQC spectra of $\mathrm{hV} 1-\mathrm{S} 1 \mathrm{~S} 4$ were collected at temperatures ranging from 15 to $50^{\circ} \mathrm{C}$ (Supplementary Fig. 6). The hV1-S1S4 resonance assignments at $45^{\circ} \mathrm{C}$ (Supplementary Fig. 3) were used to assign the temperature series spectra by chemical shift mapping, resulting in assignment of 71 resonances ( $\sim 51 \%$ of the total expected resonances) across the temperature range $\left(15-50^{\circ} \mathrm{C}\right)$. The remaining resonances could not be unambiguously identified at all temperatures because of loss of resonance intensity (i.e., peak height), emergence of resonances, and/or coalescence with other resonances. The resonances that could be assigned across the temperature series show reversible two-state temperature-dependent behavior, as illustrated by representative plots of G548 (Fig. 2d) and W549 (Supplementary Fig. 6). The two-state behavior is generally associated with all resonances we can track (Supplementary Fig. 6). Peak height simulations described in the Supplementary Methods of an idealized rigid protein under similar conditions and size of the hV1-S1S4 domain are non-sigmoidal, supporting the conclusion that the sigmoidal shapes we see in the NMR temperature titrations are indicative of a temperature-dependent conformational state change (Supplementary Fig. 7). The twostate temperature-dependent behavior is similar to that observed from $\mathrm{CD}$ and fluorescence emission experiments. Fitting the 71 assigned resonances as a function of temperature to the same two-state model used for electrophysiology, CD, and fluorescence, enabled us to evaluate the $\Delta \mathrm{H}$ value for each individual resonance. Fitting these data to a Gaussian distribution results in an average thermosensitivity of $\Delta \mathrm{H}=21.2 \pm 0.1 \mathrm{kcal} \mathrm{mol}^{-1}$ and a mean $T_{50}$ (reflective of the inflection point of the sigmoid) of $40.7 \pm 0.6^{\circ} \mathrm{C}$ (Fig. 2e and Supplementary Fig. 6). The mean $\Delta \mathrm{H}$ from NMR has a similar magnitude as those observed from CD and fluorescence measurements (Fig. 2f). To ensure that the effects observed by NMR, CD, and fluorescence arise from changes in temperature and not $\mathrm{pH}$, the experiments were carried out in sodium phosphate buffer, which is relatively insensitive to temperature-induced $\mathrm{pH}$ changes. We measured the change in $\mathrm{pH}$ to be $\sim 0.1$ units when the temperature of the buffer is varied from 10 to $60^{\circ} \mathrm{C}$ (Supplementary Table 2). Also, hV1-S1S4 ${ }^{1} \mathrm{H}$ -
${ }^{15} \mathrm{~N}$ TROSY-HSQC NMR spectra show no detectible chemical shift perturbation over this $\mathrm{pH}$ range, between $\mathrm{pH} 6.5$ and 6.4 (Supplementary Fig. 6). Taken together, the far-UV CD, intrinsic tryptophan fluorescence, and solution NMR data indicate that the hV1-S1S4 domain undergoes a temperature-dependent conformational change with a significant thermosensitivity of $\sim 20 \mathrm{kcal}$ $\mathrm{mol}^{-1}$.

Insights into temperature-dependent conformational change. The secondary structure of the hV1-S1S4 at $45^{\circ} \mathrm{C}$ was determined from experimental backbone chemical shift data analyzed in TALOS-N (Fig. 1b) ${ }^{39}$. Comparison of secondary structures of the isolated hV1-S1S4 in solution and at elevated temperature with those of the truncated rat TRPV1 structures from cryo-EM ${ }^{19,20}$ indicate that the transmembrane topologies are generally consistent (Fig. 1b). Despite the similarities, there are differences in helicity between the NMR-determined secondary structure of hV1-S1S4 at $45^{\circ} \mathrm{C}$ and the low-temperature cryo-EM structures (Fig. 3). The NMR data at $45^{\circ} \mathrm{C}$ indicate that the S2, S3, and S4 helices have short helical kinks that are absent from the TRPV1 cryo-EM structures. Among the cryo-EM structures there are also differences; for example, in the apo structure (PDB ID: 5IRZ) ${ }^{21}$, which is the putative resting state structure, the S2 helix begins near R474. However, in the putative ligand-bound structures (PDB ID: 5IS0 and 5IRX) ${ }^{21}$ the extracellular side of the S2 helix is about two helical turns longer and begins near residue K468. The NMR data at elevated temperature indicates that the S2 helical conformation reflects that of the ligand-bound structures. The NMR data also identify distinct helical content in the S2-S3 linker region, which is at the heart of the vanilloid binding pocket. In the apo- and antagonist-bound cryo-EM structures (PDB IDs: 5IRZ and 5IS0, respectively), the S2-S3 linker adopts a short amphipathic helix that includes residues S505-S510 with a break prior to the start of the S3 helix. Whereas, in the agonist activated structure (PDB ID: 5IRZ), the S2-S3 linker is not helical. The NMR-determined secondary structure is distinct and shows a near-continuous helix that begins near S502 in the S2-S3 linker and continues through the S3 helix. Additionally, the rTRPV1 structures show that the intracellular side of the S4 helix adopts a $3_{10}$ helical conformation (Fig. 1b) ${ }^{21}$. In solution at elevated temperatures, the bottom of S4 helix does not adopt a $33_{10}$ helix but instead has decreased helical probability, most similar to the agonist bound S4 helix (Fig. 1b).

To further investigate and validate the apparent differences in helicity as a function of temperature, we analyzed the amide proton chemical shifts $\left(\Delta \delta_{\mathrm{HN}}\right)$ at $45^{\circ} \mathrm{C}$ (Fig. 3a). The proton $\Delta \delta$ values were calculated by taking the difference between an assigned resonance ${ }^{1} \mathrm{H}$ chemical shift and the same amino acid in a random coil conformation. $\Delta \delta_{\mathrm{HN}}$ data typically show a characteristic periodicity in helical regions ${ }^{40-42}$. Changes in $\Delta \delta_{\mathrm{HN}}$ were plotted against the hV1-S1S4 residue numbers. At $45^{\circ} \mathrm{C}$, each helix can be fit to a sinusoidal function for $\alpha$-helices comprising the following amino acids: 419-430 for pre-S1, 439-456 for S1, 470-487 for S2 $\alpha, 490-496$ for S2 $\beta, 505-510$ for S2-S3 amphipathic loop, 511-522 for S3 $\alpha, 523-527$ for S3 $\beta$, 534-546 for S4 $\alpha$, and 547-553 for S4 $\beta$. These data for hV1-S1S4 at elevated temperature are consistent with the secondary structure output from TALOS-N. Additionally, the $\Delta \delta_{\mathrm{HN}}$ data at $45^{\circ} \mathrm{C}$ suggest that the intracellular side of the S4 helix, including residues L553 to G558, is not helical at higher temperatures. This is distinct from the consensus $3_{10}$-helix identified over this region in the cryo-EM rTRPV1 structures. The RMSE values for corresponding helices are 0.26, 0.49, 0.18, $0.15,0.33,0.27,0.22,0.12$, and 0.19 (Fig. 3a). Helicity can also be determined from NMR residual dipolar coupling (RDC) 

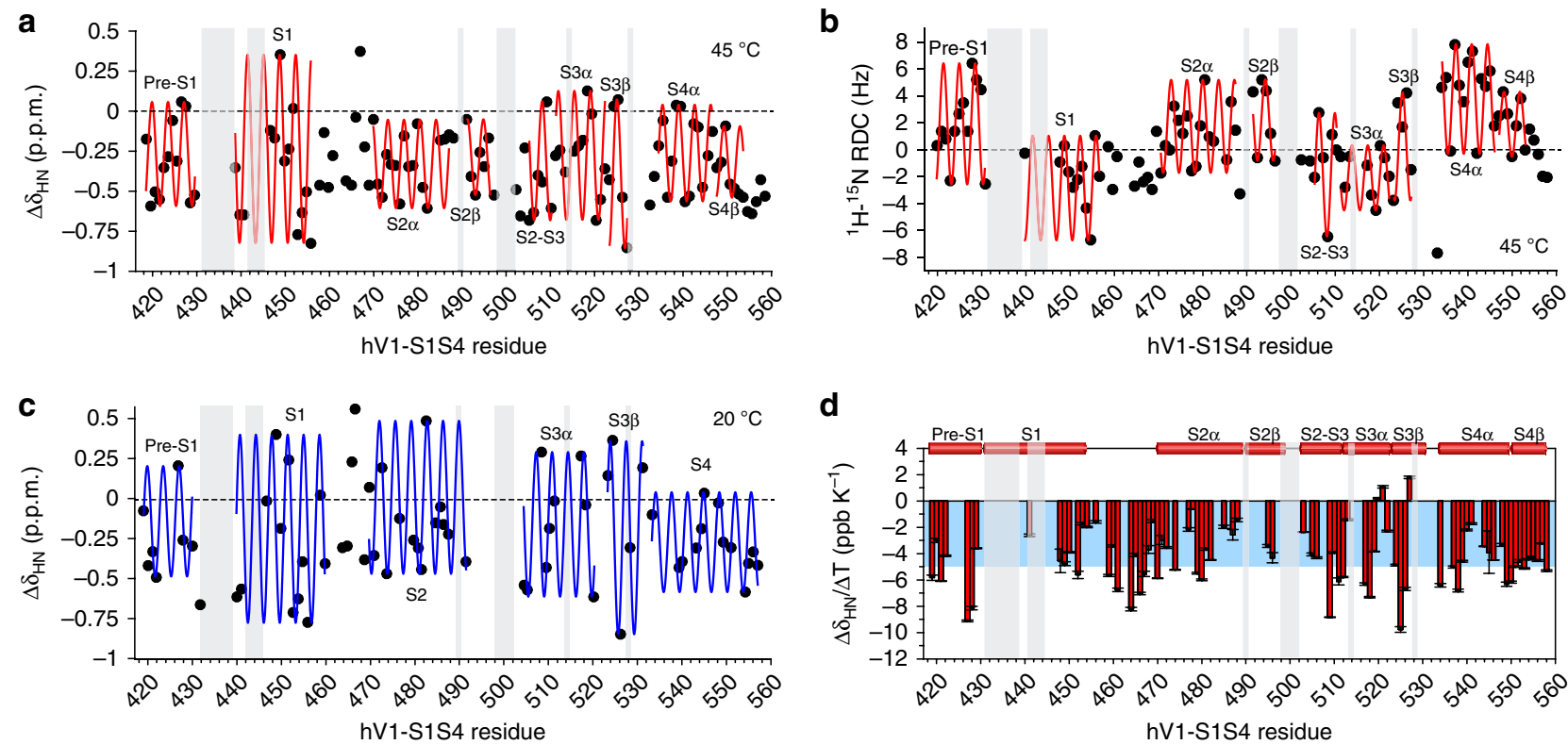

Fig. 3 hV1-S1S4 undergoes subtle temperature-induced changes in secondary structure. a Amide proton $\Delta \delta$ analysis identifies hV1-S1S4 secondary structure at elevated temperature $\left(45^{\circ} \mathrm{C}\right)$. b Dipolar wave analysis from RDC measurements of the hV1-S1S4 at $45^{\circ} \mathrm{C}$ independently identifies secondary structure. c Amide proton $\Delta \delta$ analysis at $20^{\circ} \mathrm{C}$ identifies that the hV1-S1S4 secondary structure is distinct from that at elevated temperature and generally consistent with cryo-EM secondary structures. In panels a-c, black circles are individual data points and are superimposed with fits of the data to an $\alpha-$ helical periodicity (red or blue lines respectively). d A bar plot of the amide proton temperature coefficients $\left(\Delta \delta_{\mathrm{HN}} / \Delta T\right)$ against the hV1-S1S4 residue number. Values greater than $-4.6 \mathrm{pp} \mathrm{K}^{-1}$ are indicative of hydrogen bonding and are highlighted (blue). Light gray boxes indicate regions where NMR resonances are unassigned at $45^{\circ} \mathrm{C} . \Delta \delta_{\mathrm{HN}} / \Delta T$ errors bars arise from fitting uncertainty. Source data are provided as a Source Data file.

measurements, where fitting of the data to a helical sinusoid model can be used to identify alpha-, $3_{10^{-}}$, and pi helices. This dipolar wave analysis of RDC data can also differentiate from ideal and kinked helices ${ }^{43}$. ${ }^{1} \mathrm{H}-{ }^{15} \mathrm{~N}$ RDCs were measured for hV1S1S4 at $45^{\circ} \mathrm{C}$ and used in dipolar wave analysis (Fig. 3b and Supplementary Fig. 8). The same helices used in the proton $\Delta \delta$ analysis were fit to the same sinusoidal function and the RMSE values $(\mathrm{Hz})$ for these fittings are 2.85, 2.51, 2.08, 1.18, 1.06, 1.31, $2.84,2.43,0.69$, and 1.42 . The outcomes of the RDC data further validate the TALOS-N and $\Delta \delta_{\mathrm{HN}}$ chemical shift-based secondary structure identified for the isolated hV1-S1S4 domain in solution at elevated temperatures.

In RDC data, helical distortions can be identified by changes in dipolar wave amplitudes, which are a manifestation of alignment tensor magnitude. Using this metric, the $\mathrm{RDC}$ data indicate that hV1-S1S4 in solution has helical distortions in S2, S3, and S4 transmembrane helices consistent with the chemical shift data (Fig. 3a). RDC analysis of the intracellular S4 helix confirms a lack of helicity at elevated temperature consistent with the $\Delta \delta_{\mathrm{HN}}$ data. We note that this region of the $\mathrm{S} 4$ segment at $45^{\circ} \mathrm{C}$ in both the RDC and $\Delta \delta_{\mathrm{HN}}$ data, beginning from L553, do not fit well to any helical subtype, including $\alpha-, 3_{10^{-}}$, and $\pi$ helices suggesting that this region in solution at elevated temperatures is distinct from that in the rTRPV1 cryo-EM structures. Further analysis of the experimental RDC values, and those predicted from cryo-EM structures of the rTRPV1 and mTRPV3 S1S4 domains, indicate that the general protein fold is retained. The quantitative outcomes of these experimental-predicted comparisons are detailed in Supplementary Table 3 and a visual representation shown in Supplementary Fig. 9. The correlation between cryo-EM structure derived and experimental NMR RDC values are relatively low. The origins of these correlation values likely arise from a variety of factors. Including that experimental RDC values are exquisitely sensitive to protein structure and dynamics. Generally, high correlation between experimental and predicted
$\mathrm{RDC}$ values is limited by lack of structure resolution required to predict RDC values and not from the experimental RDC accuracy $^{44}$.

Given that to date, all TRPV channel structures are $\geq 3 \AA$ resolution, one would expect a quality $(Q)$ factor beyond $40 \%$ and Pearson's correlation $(R)$ value of less than $0.9^{45}$. The lack of high-resolution structures is further exacerbated by the need to computationally add amide protons to the cryo-EM structures and account for residue insertions in the S1-S4 domain of rTRPV1 and mTRPV3 relative to hV1-S1S4. Given these challenges, and the noted discrepancies between apparent S1-S4 domain motions in the TRPV family ${ }^{18}$, the experimental and predicted correlations seem reasonable. As such the correlations should be interpreted cautiously beyond the idea that in solution, the isolated hV1-S1S4 domain structurally resembles that found in the cryo-EM structures.

In addition to the secondary structural studies at elevated temperature, we also characterized the $\Delta \delta_{\mathrm{HN}}$ values at $20^{\circ} \mathrm{C}$ (Fig. 3c). For these measurements there are fewer resonance assignments available; nonetheless, we detect significant differences in helicity at low temperature. Among these differences are that the transmembrane helix breaks are missing at $20^{\circ} \mathrm{C}$ in S2 and S4. Also, the S4 helix fits to a continuous helix. We note that this secondary structure at low temperature is generally more consistent with the rTRPV1 cryo-EM topologies, as identified from DSSP algorithm ${ }^{46,47}$, than that at elevated temperature. Taken together these studies indicate that among the structural differences between S1S4 conformational states are changes in helicity and introduction of transmembrane helix distortions.

Amide proton chemical shifts $\left(\delta_{\mathrm{HN}}\right)$ have a characteristic temperature dependence. The relationship between $\delta_{\mathrm{HN}}$ and temperature is fit to a linear function where the slope of the fit is called the temperature coefficient $\left(\Delta \delta_{\mathrm{HN}} / \Delta T\right.$, Supplementary Fig. 10). $\Delta \delta_{\mathrm{HN}} / \Delta T$ values are an indicator of the probability of hydrogen bond formation and generally reflect secondary structure 
in helical membrane proteins ${ }^{48,49}$. More specifically, residues that form intramolecular hydrogen bonds typically have $\Delta \delta_{\mathrm{HN}} / \Delta T$ values that are higher than $-4.6 \mathrm{ppb} \mathrm{K}^{-148}$. The temperature coefficients of the hV1-S1S4 were calculated and plotted against the residue number (Fig. 3d). Transmembrane helices had an average $\Delta \delta_{\mathrm{HN}} / \Delta T$ value of $-3.8 \pm 0.3 \mathrm{ppb} \mathrm{K}^{-1}$, indicating a more structured, hydrogen-bonded state; whereas, the extracellular/intracellular loops had a more negative average $\Delta \delta_{\mathrm{HN}} / \Delta T$ value of $-5.2 \pm$ $0.5 \mathrm{pp} \mathrm{K}^{-1}$ which is indicative of a lack of hydrogen bonding. We note that amide proton residual chemical shift analysis ${ }^{50}$ indicates that the majority of the resonances used in $\Delta \delta_{\mathrm{HN}} / \Delta T$ have slight non-linearity $\left(<5 \mathrm{ppb} \mathrm{K}^{-1}\right)$ that is consistent with the other NMR, $\mathrm{CD}$, and fluorescence data that shows the hV1-S1S4 undergoes a temperature-dependent conformational change (see Methods and Supplementary Fig. 10).

Coupling the secondary structure analysis of the hV1-S1S4 domain from TALOS-N, $\Delta \delta_{\mathrm{HN}}$, and ${ }^{1} \mathrm{H}_{-}{ }^{15} \mathrm{~N}$ RDCs, with $\Delta \delta_{\mathrm{HN}} /$ $\Delta T$ and $\mathrm{D} / \mathrm{H}$ exchange factor data (Fig. 1b, c, and Fig. 3) shows that in solution at elevated temperatures this domain retains the expected transmembrane topology, but with particular changes in helicity that either generally reflect the agonist activated cryo-EM structure or are distinct from the rTRPV1 cryo-EM structures.

To probe the magnitudes and directions of conformational change detected in the temperature titration studies of hV1-S1S4, NMR-detected PRE distance measurements were made from proton relaxation experiments at 20 and $50^{\circ} \mathrm{C}$. These experiments exploit the lone endogenous cysteine residue (C443, S1 helix) in hV1-S1S4 for MTSL labeling, obviating the need to engineer mutations that might impact the potential temperaturedependent structural changes. To ensure the highest possible accuracy of these measurements, six separate samples were prepared, the paramagnetic (three samples) and diamagnetic species (three samples) were each recorded in triplicate at each temperature. To accurately convert the PRE data to distance information, the rotational correlation times $\left(\tau_{c}\right)$ were also directly measured at both temperatures using TRACT (TROSY for rotational correlation times) NMR experiments ${ }^{51}$. Using exclūsively the W549 indole amine resonance, which is membrane embedded in the middle of the S4 transmembrane helix, the hV1-S1S4 rotational correlation times at 20 and $50{ }^{\circ} \mathrm{C}$ were calculated to be $49 \pm 3$ and $25.6 \pm 0.1 \mathrm{~ns}$, respectively (Supplementary Fig. 8).

Distances from the residues of the hV1-S1S4 to MTSL-labeled C443 (Supplementary Fig. 8) were obtained at 20 and $50^{\circ} \mathrm{C}$. From these data, the differences in PRE distances between 20 and $50^{\circ} \mathrm{C}$ were calculated $\left(\triangle \mathrm{PRE}_{50}{ }^{\circ} \mathrm{C}-20^{\circ} \mathrm{C}\right)$ and these values were plotted as a function of residue number (Fig. $4 \mathrm{a}$ ). Given the magnitudes of the temperature-dependent changes in distance, and the proclivity of PRE data to encode both structural and dynamic information, we interpret these data in a qualitative manner. The sign of the $\triangle \mathrm{PRE}$ values indicates that a given amide proton is moving towards (negative value) or away (positive value) from MTSL-C443. The $\triangle \mathrm{PRE}_{50}{ }^{\circ} \mathrm{C}-20^{\circ} \mathrm{C}$ suggest temperature-driven changes in dynamics or conformational states in the S1-S2 loop, the S2-S3 loop, and the S4 helix C-terminus. The S1-S2 loop has complex data trends suggestive of differences in loop dynamics between temperatures. The S2-S3 loop, which contains key residues in the vanilloid binding site, and S4 $\beta$ helix C-terminus show general trends of movements away from C443. Precise interpretation on these temperature-dependent PRE changes is challenging; however, we note that the S1-S2 loop, the S2-S3 loop, and the S4 C-terminus, have been implicated in proton activation ${ }^{52}$, ligand binding ${ }^{20,23}$, and ligand coupling ${ }^{53}$, respectively and is consistent with observations of allosteric coupling of TRPV1 activation modes.

${ }^{15} \mathrm{~N}$-edited NOESY-TROSY measurements provide throughspace distance information between protons up to distances of $\sim 5$ Å. NOESY data also provide a measure of solvent exposure via NOE cross-peaks between a given backbone amide proton resonance $\left(\mathrm{H}_{\mathrm{N}}\right)$ and the water resonance $(\sim 4.7 \mathrm{ppm})$. Since the $\mathrm{H}_{\alpha}$ to $\mathrm{H}_{\mathrm{N}}$ distances represent a fixed distance, taking the intensity ratios between the water- $\mathrm{H}_{N}$ cross-peak and the $\mathrm{H}_{\alpha}-\mathrm{H}_{N}$ crosspeak $\left(I_{\text {water }} / I_{\mathrm{Ha}}\right)$ should reflect a normalized measure of solvent accessibility of a specific backbone amide resonance. Furthermore, taking the difference between the normalized ratios at high and low temperatures $\left(\Delta \mathrm{NOE}_{45}{ }^{\circ} \mathrm{C}-20^{\circ} \mathrm{C}\right)$ identifies which residues change solvent exposure as a function of temperature. With this in mind, a positive (negative) $\triangle \mathrm{NOE}_{45}{ }^{\circ} \mathrm{C}-20^{\circ} \mathrm{C}$ indicates increased (decreased) solvent exposure at $45^{\circ} \mathrm{C}$. NOE-based solvent exposure between elevated and lower temperature indicate that for the vast majority ( $60 \%$ of the observed residues) of $\mathrm{hV} 1$ S1S4, the changes in hydration are minimal. However, the S1-S2, S2-S3, and the S3-S4 loops have increased solvent exposure at higher temperatures. Similarly, the S4 helix C-terminus also becomes more solvent-exposed at higher temperatures (Fig. $4 \mathrm{~b}$ and Supplementary Fig. 11). The temperature-dependent changes in secondary structure, distance, and solvation are schematized in Fig. $4 c$.

R557 couples the S1-S4 domain to the pore domain. Our data show that the C-terminus of the S4 helix partially unwinds and becomes more solvent-exposed at elevated temperatures. These movements include the conserved R557, as monitored from the backbone amide with PRE, RDC, chemical shift, and NOESY NMR data. A previous study indicated that R557 in rat TRPV1 is involved in coupling the TRPV1 S1-S4 domain to channel activation in part via a cation $-\pi$ interaction rearrangement between Y554 and R557 that impacts diverse activation mechanisms, including thermosensing ${ }^{53}$. Our biophysical data studies tentatively support this coupling mechanism. To probe the role of human R557-TRPV1 in temperature gating, we generated a human TRPV1-R557A mutation for electrophysiology studies. Whole-cell patch-clamp measurements of hTRPV1-R557A corroborate that this mutation inhibits TRPV1 thermosensitivity (Fig. 5a, b) and using NMR we show that for hV1-S1S4 the R557water NOE cross-peak is absent at $20^{\circ} \mathrm{C}$ and present at $45^{\circ} \mathrm{C}$ (Fig. 5c). These data indicate that R557 in the S4 helix undergoes a temperature-dependent change in solvent exposure that is consistent with the $\triangle \mathrm{PRE}$ measurements. Using NMR temperature titrations with hV1-S1S4-R557A mutant (Supplementary Fig. 12), the resonance intensities retained the sigmoidal two-state temperature dependence as shown in the representative plot of G548, with a $\Delta \mathrm{H}$ of $19 \pm 2 \mathrm{kcal} \mathrm{mol}^{-1}$ (Fig. 5d). Measuring 70 resonance intensities as before with WT, the mean $\Delta \mathrm{H}=19.7 \pm$ $0.1 \mathrm{kcal} \mathrm{mol}^{-1}$ and the mean $T_{50}=39 \pm 2{ }^{\circ} \mathrm{C}$ (Fig. 5e and Supplementary Fig. 12). We attribute the small decrease in thermosensitivity $\left(\Delta \Delta \mathrm{H}=1.5 \pm 0.1 \mathrm{kcal} \mathrm{mol}^{-1}\right)$ between the wild-type and R557A mutant of hV1-S1S4 to the loss of the cation- $\pi$ interaction between R557 and Y554. Comparison of the WT and R557A hV1-S1S4 ${ }^{1} \mathrm{H}_{-}{ }^{15} \mathrm{~N}$ TROSY-HSQC spectra show that Y554 undergoes significant chemical shift perturbation consistent with the loss of the cation $-\pi$ interaction (Fig. 5f). If R557 couples the S1-S4 domain with the PD, then the R557A mutation should also knock-out capsaicin activation. Whole-cell patch-clamp electrophysiology of human TRPV1-R557A shows that this mutation indeed silences capsaicin activation (Supplementary Fig. 12) while capsaicin binding studies of the R557A hV1-S1S4 domain with NMR show that the mutant retains the ability to bind capsaicin with the same affinity as WT (Supplementary Fig. 12). The fact that R557A ablates temperature and capsaicin sensitivity begs the question if it is a functional channel. Previous studies of TRPV1 suggest that the pore domain is key to proton 


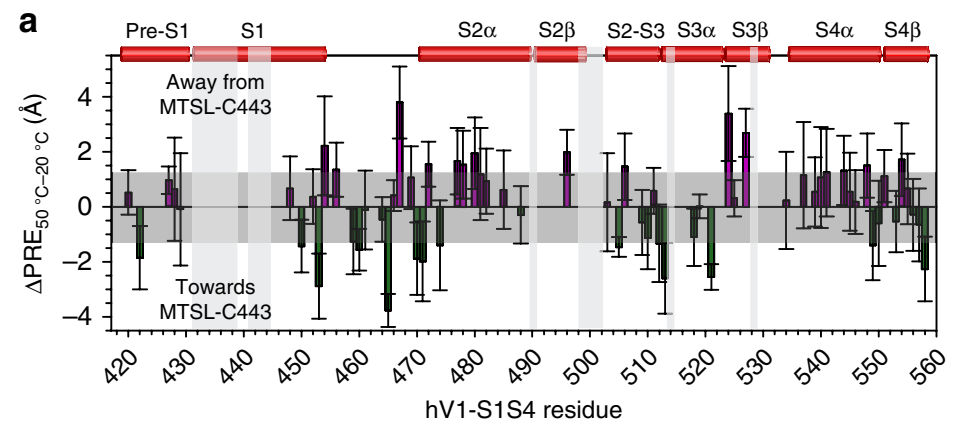

c
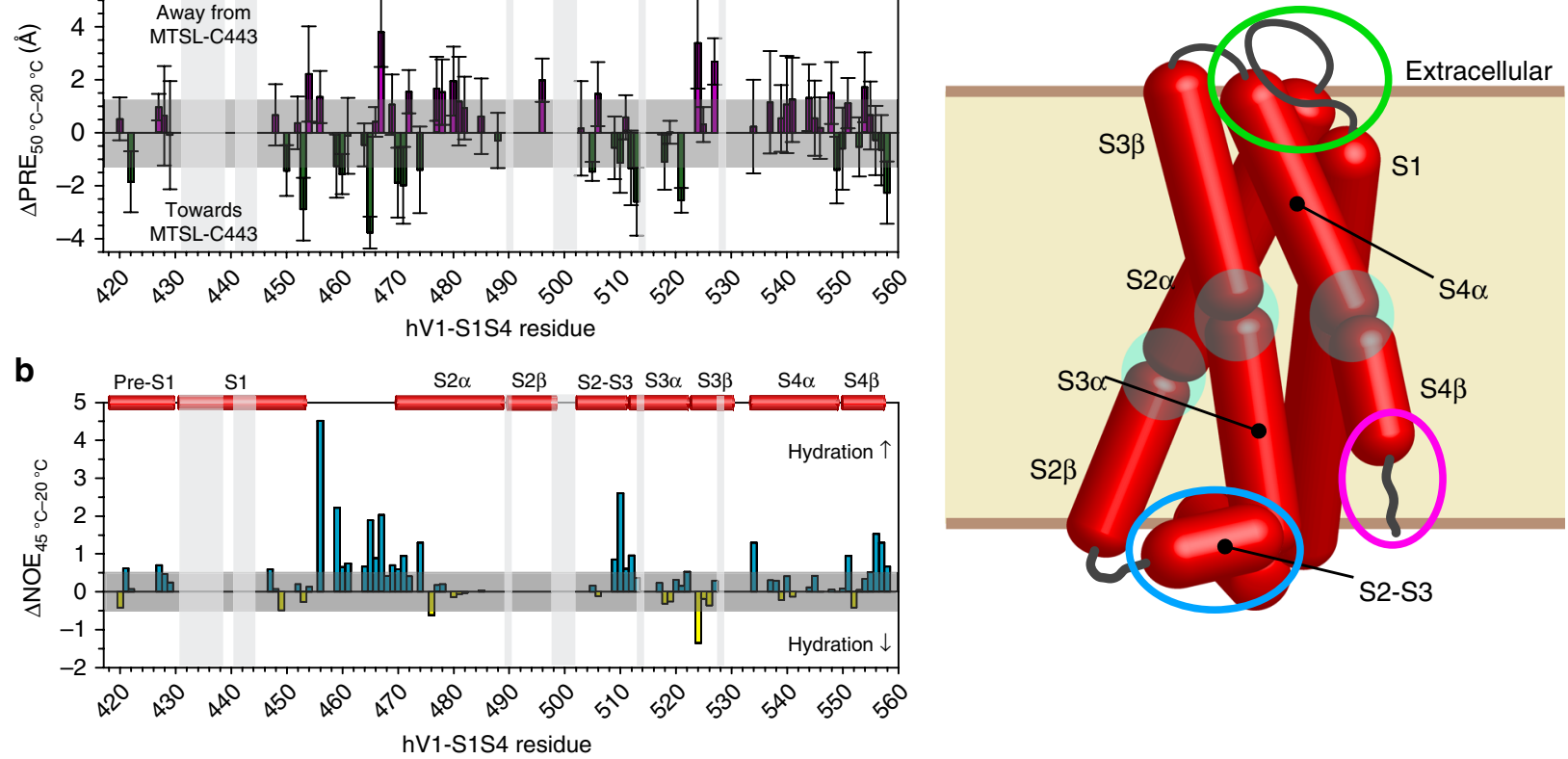

Fig. 4 hV1-S1S4 undergoes discrete temperature-induced changes in distances, and solvent accessibility. a Temperature-dependent differences in distances measured using paramagnetic relaxation enhancement ( $\triangle \mathrm{PRE}$ ) of the hV1-S1S4 at 20 and $50{ }^{\circ} \mathrm{C}$. The average of the s.e.m, $1.2 \AA$, was used as a threshold value and shown in gray. Errors are propagated s.e.m. $\mathbf{b}$ Changes in hydration as a function of temperature were measured from normalized $\mathrm{H}_{\mathrm{N}}$-water resonance intensities from ${ }^{15} \mathrm{~N}-\mathrm{NOESY}$-TROSY data at 20 and $45^{\circ} \mathrm{C}$. The $\Delta \mathrm{NOE}_{45}{ }^{\circ} \mathrm{C}-20^{\circ} \mathrm{C}$ is reflective of changes in hydration where loops and the bottom of S4 helix exhibit increased hydration at elevated temperatures. c A cartoon of hV1-S1S4 identifying regions that change as a function of temperature. Blue and magenta circles indicate increased and decreased helicity, respectively. The green circle highlights differences in the S1-S2 loop solvent accessibility. Transmembrane helix distortions are highlighted as transparent cyan circles. The blue highlighted region shows helical differences in the vanilloid binding site and the magenta identifies the C-terminus of the S4 helix which becomes more solvent exposed and less helical at elevated temperatures. Source data are provided as a Source Data file.

activation $^{54,55}$; if R557A is an S1S4 domain-coupling mutation, we would anticipate that $\mathrm{pH}$ activation would be unaffected. Functional measurements of R557A hTRPV1 show that the mutant channel retains $\mathrm{pH}$ sensitivity with a proton $\mathrm{EC}_{50}$ of $\sim 5.7$, which is on par with the WT hTRPV1 channel (Supplementary Fig. 13) 52,56 . The conclusion that R557 functions to couple motions of the hV1-S1S4 domain to channel gating is supported by relatively high thermosensitivity and the retention of capsaicin binding of the isolated R557A hV1-S1S4 domain, with the corresponding functional loss of temperature and capsaicin activation for the full-length R557A hTRPV1.

\section{Discussion}

The ability to sense and respond to temperature is fundamental in biology, with a variety of temperature-sensing requirements that vary from low to high temperatures and acute (fast) to environmental (sustained) responses. Acute hot-sensing in higher organisms is sufficiently crucial that the pathway is triply redundant with three distinct heat receptors; the best studied of these is TRPV $1^{57}$. Similarly, TRPV1 thermosensitivity is tuned in a highly species-dependent manner that is correlated with environmental niche. For example, desert dwelling species that are exposed to sustained elevated temperatures are thought to generally have attenuated heat-sensing responses ${ }^{58}$. Many factors have been shown to modulate the temperature response of TRPV1, including chemical ligands, $\mathrm{pH}$, lipids, and proteins. Nonetheless, TRPV1 is intrinsically thermosensitive and the ability to integrate changes in biologically relevant temperatures as a molecular thermometer is a feature inherent to the protein ${ }^{34}$.
Despite its importance, the mechanism of TRPV1 thermosensing has been complicated by seemingly contradictory outcomes ${ }^{1,2}$.

In this study, we evaluate the conformational change and thermodynamics of an isolated human TRPV1 S1-S4 domain. We establish that the S1-S4 domain is stable, retains the expected membrane topology and structure, and recapitulates anticipated capsaicin agonist binding, indicating the isolated domain resides in a biologically relevant conformation. These results are consistent with structurally homologous domains studied in isolation from other TRP ${ }^{59}$, sodium ${ }^{60}$, potassium ${ }^{42,61-63}$, and proton ${ }^{64}$ ion channels.

Temperature-dependent studies of the hV1-S1S4 monitored by $\mathrm{CD}$, intrinsic tryptophan fluorescence, and solution NMR show that this domain undergoes a two-state temperature-dependent transition, with significant thermosensitivity ( $\Delta \mathrm{H}$ of $\sim 20 \mathrm{kcal} \mathrm{mol}^{-1}$ ). These data also indicate that the domain retains significant structure in both high and low temperature conformations. The energetics associated with this conformational change are large in comparison to other proteins. For example, the enthalpy magnitude of the hV1-S1S4 conformational transition is about twice as large as the well-studied tetrameric hemoglobin system, despite the size of hemoglobin being about four-times larger by mass ${ }^{29}$.

Putting hV1-S1S4 thermosensitivity value into the context of the full-length TRPV1 channel will require future studies. The thermosensitivity, or change in enthalpy, between temperature resting and active states of full-length TRPV1 has a consensus value of $\sim 100 \mathrm{kcal} \mathrm{mol}^{-1}$ (Supplementary Table 1 ). The value measured here for the full-length human TRPV1 heterologously expressed in mammalian cells is $98 \pm 12 \mathrm{kcal} \mathrm{mol}^{-1}$ or $94 \pm 8 \mathrm{kcal}$ $\mathrm{mol}^{-1}$, depending on the method used. One challenge with 
a
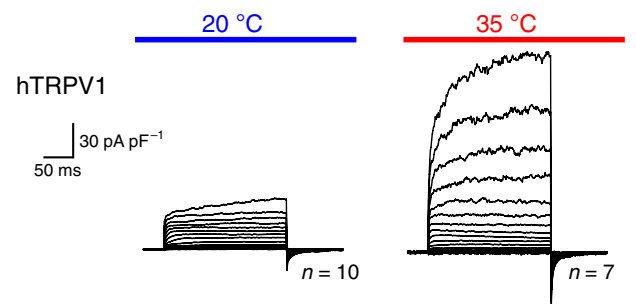

hTRPV1-R557A
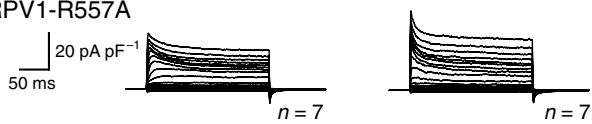

b

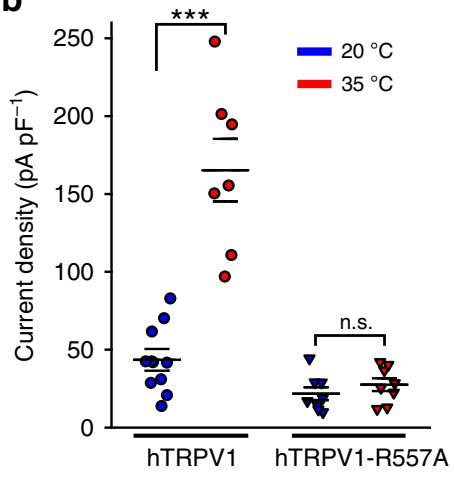

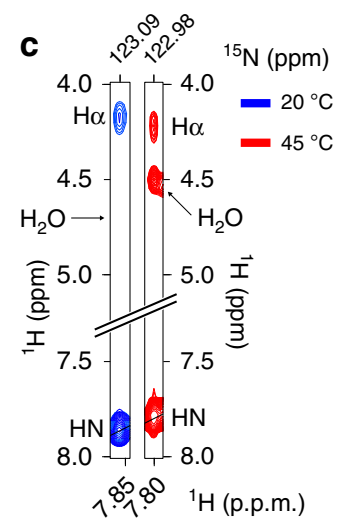

d

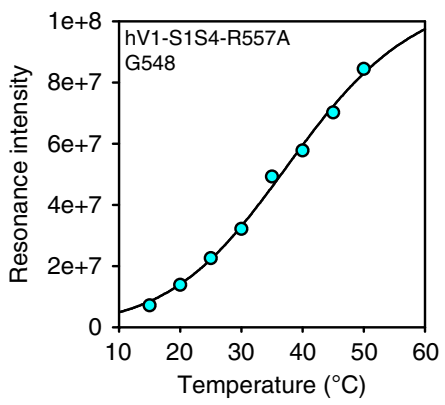

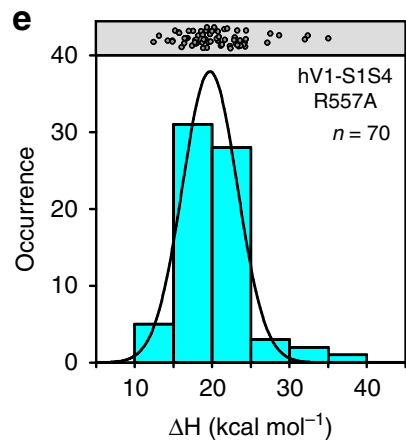

f

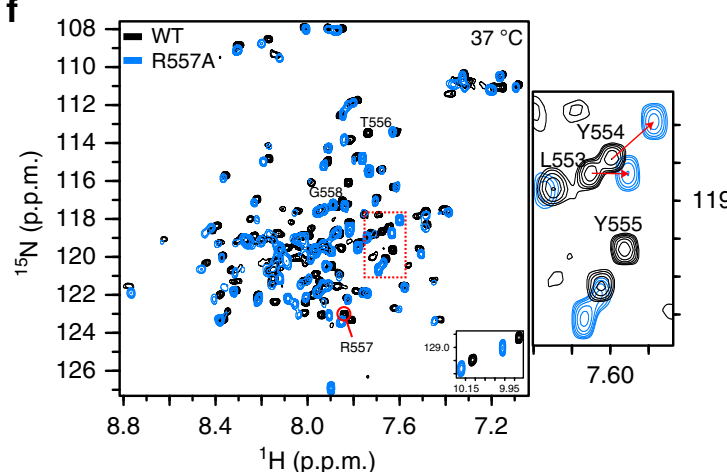

Fig. 5 TRPV1 R557 is a crucial residue for coupling between the S1-S4 and pore domains. a, b Whole-cell patch-clamp electrophysiology data from HEK293 cells show that the wild-type hTRPV1 is heat activated, while the hTRPV1-R557A mutant is not. Error bars in panel (b) are s.e.m. c ${ }^{15} \mathrm{~N}$-edited NOESY-TROSY data of the R557-water resonance cross-peak is missing at low temperature but present at elevated temperature, suggesting that R557 amide backbone moves from a membrane embedded to a solvent-accessible position in a temperature-dependent manner. $\mathbf{d}$ NMR temperature titration of hV1-S1S4-R557A. G548 in hV1-S1S4-R557A resonance intensity as a function of temperature shows two-state behavior analogous to the wild-type hV1S1S4 domain (compare Fig. 2d). e A histogram and a Gaussian distribution fit of $\Delta \mathrm{H}$ identify a mean $\Delta \mathrm{H}=19.7 \pm 0.1 \mathrm{kcal} \mathrm{mol}^{-1}$ for the mutant hV1-S1S4R557A ( $n=70$ residues). The data are suggestive of a temperature-dependent conformational change in hV1-S1S4-R557A. $\mathbf{f}$ Comparison of ${ }^{1} \mathrm{H}-15 \mathrm{~N}$ TROSY-HSQC spectra show that the R557A mutation causes perturbation at Y554 supporting that the R557A mutation disrupts the cation- $\pi$ interaction. Source data are provided as a Source Data file.

interpreting the thermosensitivity of the isolated hV1-S1S4 relative to the tetrameric TRPV1 in biological conditions is that the cooperativity between monomers during heat-sensing and activation in TRPV1 remains unclear. hV1-S1S4, which represents $\sim 18 \%$ of the channel by mass, could potentially contribute the majority of the thermodynamic driving force $(>80 \%)$ should the $\Delta \mathrm{H}$ values of the isolated hV1S1-S4 be additive (noncooperative) in nature. On the other hand, if S1-S4 domains allosterically couple very efficiently (fully cooperative) in tetrameric channel state, then this domain might contribute as little as $\sim 20 \mathrm{kcal} \mathrm{mol}^{-1}$, or $\sim 20 \%$ of the thermosensing driving force, to TRPV1 thermosensitivity.

Nonetheless, our results fit in well with the existing literature with the thermodynamic and mechanistic information about TRPV1 and thermosensing in general ${ }^{18,32,36,65}$. For example studies of the temperature-sensitive $\triangle \mathrm{N}$-TRPV1, a TRPV1 isoform, that lacks the majority of the $\mathrm{N}$-terminus indicates that the core TRPV1 temperature sensing region resides in the transmembrane domain ${ }^{35}$. Our data and a chimeric study of the Shaker voltage-gated potassium channel $(\mathrm{Kv1.2})$ with the rat TRPV1 pore domain (S5-S6 helices, residues 575-687) suggest that both membrane domains likely contribute to thermosensing. In the chimeric study, the TRPV1 PD was sufficient to transform the chimeric Shaker channel into a heat activated channel with a thermosensitivity of $\sim 75 \mathrm{kcal} \mathrm{mol}^{-1}$ as estimated from the reported $Q_{10}$ value ${ }^{36}$. Clear interpretation of these $P D$ chimeric studies are complicated by voltage-gated potassium channel temperature sensitivity ${ }^{66}$. Further studies are required to better contextualize our thermodynamics studies with regards to the full-length channel and to decipher and quantify the cooperativity between membrane domains. If rat TRPV1 vanilloid ligand activation serves as a guide, concatemer studies have shown that a single capsaicin binding event can fully open the channel (i.e., is highly-cooperative); on the other hand, equivalent proton activation studies, show that full activation requires all four TRPV1 proton-sensors and is generally non-cooperative ${ }^{67}$.

Beyond thermodynamic contributions to TRPV1 thermosensitivity, there are two general views on the mechanisms of thermosensing; first, that state-dependent solvation changes, which have large thermodynamic signatures, drive thermosensing; or second, that partial unfolding as a function of temperature (i.e. TRPV1 is only marginally stable) gives rise to the large thermosensitivity and drives thermosensing ${ }^{1,29}$. While mechanistic TRPV1 temperature gating details continue to emerge, our studies of hV1-S1S4 domain offer particular mechanistic insights. Our NMR studies of the hV1-S1S4 at elevated temperatures identify structural differences from the cryo-EM structures. For example, chemical shift and RDC data show that the S2, S3, and S4 helices are kinked at elevated temperature, a feature absent in all of the current TRPV1 structures. However, S2 helical kinks are found in certain TRPV3, TRPV5, and TRPV6 structures ${ }^{68-71}$. Similarly, helical distortions in $\mathrm{S}^{72}$ and $\mathrm{S} 4$ are also found in other 
TRPV family structures. The most significant structural temperaturedependent changes in distance, hydration, and secondary structure from our studies identify three particular areas: (1) The extracellular juxtamembrane region of the S1 helix and S1-S2 loop, (2) the intracellular S2-S3 loop, and (3) the intracellular S4 helix. Interestingly, these temperature-induced changes we observe are similar to those identified via molecular dynamics simulations of TRPV1 heat activation ${ }^{73}$.

The extracellular C-terminus of the S1 helix and the S1-S2 loop have been implicated in proton activation, where R455 (in rTRPV1, R456 in hTRPV1) is critical to channel function and $\mathrm{R} 455 \mathrm{~K}$ causes a gain of function (GOF) mutation ${ }^{74}$. This residue in the cryo-EM structure is buried in the membrane as identified by the OPM server ${ }^{75}$ and is in close proximity to lipid molecules (Supplementary Fig. 11). Moreover, this arginine is proximal to two residues that are crucial for the proton activation, V538 in S4 helix and T633 in pore helix ${ }^{52}$. A study that engineered an RTxsensitive TRPV3 revealed that an allosteric point mutation in the pore domain $(\mathrm{A} 606 \mathrm{~V})$ along with mutations to the vanilloid binding pocket, is necessary for TRPV3 to be both RTx and temperature sensitive, emphasizing the role of this residue in temperature sensitivity and the allosteric modulation of the channel $^{76}$. In TRPV1, the equivalent residue to A606 is V596, and this residue is surrounded by R456, V538, and T633 (Supplementary Fig. 11). With this region of the S1S4 domain clearly important for function, it is interesting that our temperaturedependent NMR-studies identify meaningful changes in distance and hydration which is suggestive that TRPV1 temperature activation might partially interface with proton activation mechanisms that are coupled to the upper pore gate.

Another area identified in our NMR studies is a difference in helicity of the S2-S3 linker relative to the cryo-EM structures. The S2-S3 linker is central to vanilloid ligand activation of TRPV $1^{23,26,28}$ and includes Y511 and S512 that impart specificity by hydrogen bonding to the vanilloid head group of TRPV1 agonists. In the cryo-EM structures, there are helical differences between vanilloid bound and apo-TRPV1 structures. Our NMR data identify an additional helical turn in the S2-S3 linker (Fig. 1b, 3a, b) suggestive that temperature-dependent conformational changes of this domain interface with features key to vanilloid activation.

A relatively common feature of TRPV family structures is a stretch of $3_{10}$ helix at the intracellular side of the S4 helix. Interestingly, this is not present in our NMR investigations of the hV1-S1S4, instead we see a kinked $\alpha$-helix where the transition between $\alpha$ - and $3{ }_{10}$-helix is found in most TRPV structures. In addition, at elevated temperature we see in the RDC data that there is a loss of one helical turn at the S4 C-terminus (Fig. 3b). This loss of helix is coupled with a general movement of the S4 helix towards C443 in the S1 helix, as identified in PRE measurements (Fig. 4a), indicating a concerted movement towards the center of the membrane plane. These changes in secondary structure and distance are concomitant with an increase in hydration of the S4 C-terminus (Fig. 4b). Similar to the loss of helicity at the C-terminus end of the S4 helix in the hV1-S1S4, a modest unfurling of the bottom of the S4 helix in the activated state is found in TRPV1, TRPV2, TRPV3, and TRPM8 (Supplementary Figs. 14, 15). This helix unfurling is also shown in the apparent TRPM8 ligand-gating mechanism ${ }^{77}$. Upon an agonist binding, the TRPM8 S4 helix undergoes changes in secondary structure from an $\alpha$ - to a 310 -helix, starting around V839, pushing the membrane-bound R850 out the membrane (Supplementary Fig. 14). This R850 in TRPM8 appears to be homologous to R557 in TRPV1 as these residues are located in the same region in the membrane (Supplementary Fig. 14). Recent TRPV3 structural studies that capture the putative temperature-dependent conformational changes,

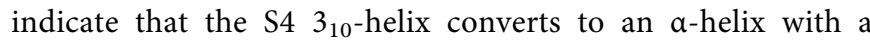
mutant that reflects the putative temperature-activated state ${ }^{78}$, similar to what our temperature-dependent studies indicate. This TRPV3 structural study also implicates changes in solvent exposure to the S1-S2 loop that causes distortions in S1, S2, and S3 helices. Similarly, there are secondary structure differences in the S2-S3 loop (Supplementary Fig. 15). Taken together, these putative temperature-dependent TRPV3 structural changes are consistent with what we identify with our solution NMR studies.

The C-terminal S4 region is functionally important in coupling the $\mathrm{S} 1-\mathrm{S} 4$ domain to the pore domain ${ }^{53}$. Our data identify that $\mathrm{R} 557$ in S4b, which is membrane-bound in the cryo-EM structures and at $20^{\circ} \mathrm{C}$ in our NOE-detected solvation studies, becomes water accessible at elevated temperatures. This residue is well-conserved in TRPV1 orthologs, and mutation to lysine (R557K) results in a GOF mutation ${ }^{53}$. The TRPV1 cryo-EM structures have provided molecular insight into the role of R557 in ligand-activated channel coupling. In the apo structure, R557 forms a cation $-\pi$ interaction with Y554 and a lipid separates R557 from E570 in the S4-S5 linker (Fig. 6a) ${ }^{21}$. Vanilloid binding releases the bound lipid, allowing for a switch in R557 conformation to interact with E570 that leads to channel activation (Fig. 6a) ${ }^{21}$. Our data indicate that heat causes similar conformational changes that would result in the disruption of the cation $-\pi$ interaction between Y554 and R557, as R557 changes helicity and becomes more solvent accessible at elevated temperatures (Fig. 6b). Presumably, these changes could couple analogously to vanilloid binding to gate the pore domain. To probe this, we generated an R557A mutant, and electrophysiology studies show that it has both impaired heat and capsaicin sensitivities, while retaining proton activation (Fig. 5 and Supplementary Figs. 12, 13), consistent with the disruption of S1-S4 domain coupling to gating. The R557A mutation was also incorporated into the isolated hV1-S1S4 and the NMR data show that it retains structure, and only modestly impacts thermosensitivity by $\sim 1.5 \mathrm{kcal} \mathrm{mol}^{-1}$ relative to the wild-type (Supplementary Fig. 12). Presumably this slight loss of thermosensitivity is from the oblation of the Y554-R557 cation- $\pi$ interaction. In TRPV4, the residues equivalent to Y554 and R557 in TRPV1 (Y591 and R594) have also been implicated in ligand activation, and mutations in the TRPV4 S3 and S4 helices also impacts heat activation ${ }^{79}$.

The TRPV1 paralog TRPV3 is heat (warm) activated but is not natively sensitive to vanilloid activation. However, vanilloid sensitivity has been engineered to the potent vanilloid resiniferatoxin (RTX), suggesting a mechanistic overlap between TRPV1 and TRPV3 ${ }^{76}$. The residue equivalent to Y554 in TRPV3 is Y564 (Supplementary Fig. 15) and functional characterization of TRPV3 Y564A shows that this channel is highly sensitized relative to the WT TRPV3 and reduces thermosensitivity, as estimated from the reported $Q_{10}$ values, from a $\Delta \mathrm{H}$ of $\sim 65 \mathrm{kcal} \mathrm{mol}^{-1}$ for the WT channel to $\sim 4 \mathrm{kcal} \mathrm{mol}^{-1}$ for the Y564A mutant ${ }^{78}$. Taken together the TRPV1, V3, and V4 data suggest that R557 in TRPV1 functions as a switch, which couples both heat and ligand stimuli (Figs. 5-6 and Supplementary Fig. 12) to the pore domain in gating and is in agreement with a mechanism proposed by others $^{21}$. We note that R557 is not included in the construct used in a study that swapped the TRPV1 PD to the temperatureinsensitive Shaker channel (Supplementary Fig. 12) ${ }^{36}$, suggesting that in the context of the full-length TRPV1 channel, fully interpreting the role of the PD in thermosensing is complex. Our S1-S4 studies suggest that interplay between these membrane domains are required for heat activation.

Overall, our study provides insights into the role of the S1-S4 domain in TRPV1 gating. We also present direct binding measurements of capsaicin to the S1-S4 domain and validate the 


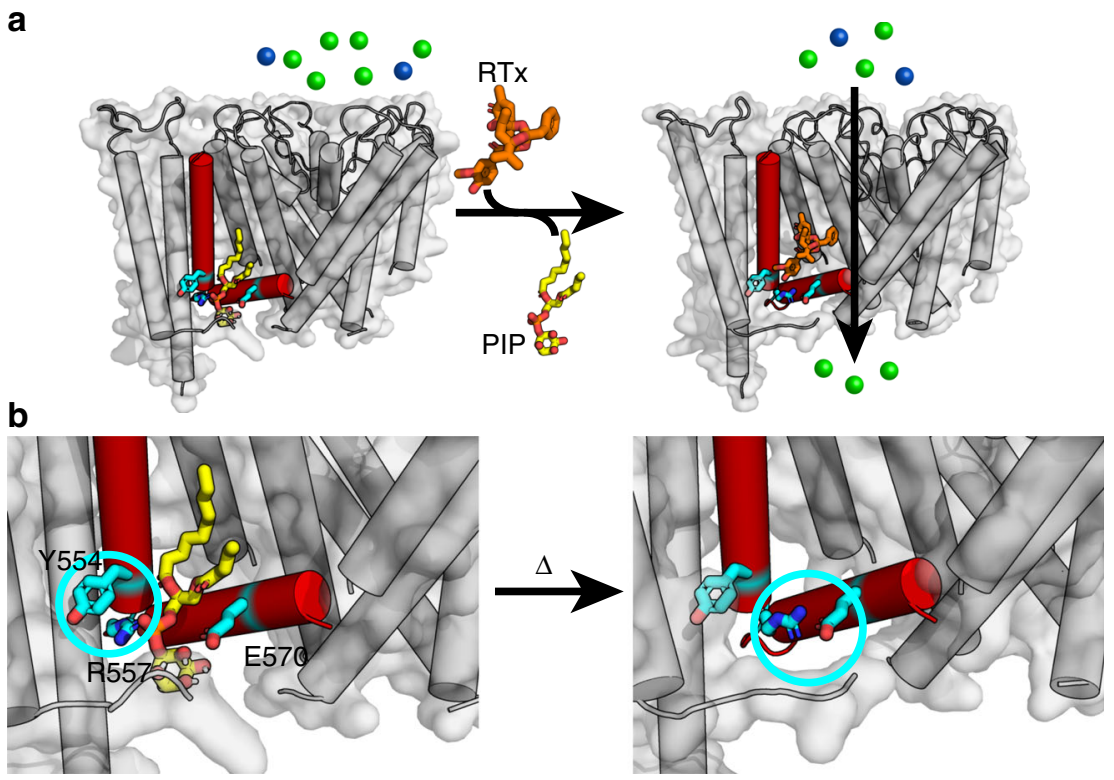

Fig. 6 A proposed heat-sensing mechanism of TRPV1 involves the interaction between the S4 helix and the S4-S5 linker. a Structure-based ligandgated mechanism of TRPV1 is shown in cartoon (PDB ID: 5IRZ and 5IRX). In the resting state, an endogenous lipid (yellow) occludes the canonical vanilloid binding pocket, perturbing the interaction between the R557 in S4 and E570 in S4-S5 linker. As a vanilloid ligand, resiniferatoxin (RTx, orange), binds the S1-S4 domain, the cation- $\pi$ interaction between R557 and Y554 is disrupted and R557 changes conformation to interact with E570, opening the lower gate. For visual clarity, S3 helix is omitted, and only one S1-S4 domain is shown. b The close-up views that show the interaction between Y554 and R557 in S4 helix in the resting state (left, highlighted in cyan circle), and the interaction between R557 and E570 in S4-S5 linker (right, shown in cyan circle). Our data suggest that the C-terminal end of the $\mathrm{S} 4$ helix undergoes heat-induced movements in this region and loses helicity. This model is analogous to the ligand activation mechanism and suggests an understanding for overlap between heat and ligand activation.

identity of key residues that exhibit specific capsaicin binding. Utilizing various techniques, we show that the S1-S4 domain undergoes a temperature-dependent conformational change with a relatively large magnitude $\Delta \mathrm{H}$ (thermosensitivity). Mechanistic studies suggest that there is overlap between regions of the S1-S4 domain that have been previously implicated in proton and ligand activation and the conformational changes identified in our study. Taken together our data indicate that the S1-S4 domain contributes to TRPV1 thermosensitivity and suggests avenues to probe the allosteric networks that regulate the polymodal activation of TRPV1.

\section{Methods}

Human hV1-S1S4 protein expression and purification. The hV1-S1S4 (S1-S4, including Pre-S1) was expressed from a synthetic gene engineered from ATUM that encodes the hV1-S1S4, a 141-residue structural domain with an identical amino acid sequence to human TRPV1. The optimized gene included an Nterminal $10 \times$ His tag fused to a thrombin cleavage site flanked by $\mathrm{NcoI}$ and BamHI restriction sites. These restriction sites were used to subclone the hV1-S1S4 into a pET16b vector. Sanger sequencing validated the integrity of the synthetic gene. All primers used in these studies are shown in Supplementary Table 4. The expressed protein includes residues Pro418-Gly558 from the human TRPV1 channel.

We optimized the fusion hV1-S1S4 expression using Escherichia coli strain BL21 (DE3) competent cells. Starter cultures were initiated with 1 transformed colony inoculated in a culture tube containing $3 \mathrm{~mL} \mathrm{LB}$ and $0.1 \mathrm{mg} \mathrm{mL}^{-1}$ ampicillin $(3 \mu \mathrm{L}$ of $100 \mathrm{mg} \mathrm{mL}^{-1}$ stock) (Sigma Aldrich) and incubated for ca. $7 \mathrm{~h}$ at $37^{\circ} \mathrm{C} .6 \mathrm{~mL}$ of starter culture was used to inoculate $1 \mathrm{~L}$ of $\mathrm{M} 9$ minimal media $(12.8 \mathrm{~g}$

$\mathrm{Na}_{2} \mathrm{HPO}_{4} \cdot 7 \mathrm{H}_{2} \mathrm{O}$ (Sigma Aldrich), $3 \mathrm{~g} \mathrm{KH} \mathrm{KO}_{4}$ (Thermo Fisher Scientific), $0.5 \mathrm{~g}$ $\mathrm{NaCl}$ (Thermo Fisher Scientific), $1 \mathrm{mM} \mathrm{MgSO} \mathrm{m}_{4} \cdot 7 \mathrm{H}_{2} \mathrm{O}$ (Sigma Aldrich), $0.1 \mathrm{mM}$ $\mathrm{CaCl}_{2} \cdot 2 \mathrm{H}_{2} \mathrm{O}$ (Sigma Aldrich), 4\% D-glucose (w/v) (Sigma Aldrich), $1 \times \mathrm{MEM}$ Vitamin solution $\left(100 \times\right.$ solution, Corning)) with $1 \mathrm{~g}^{15} \mathrm{NH}_{4} \mathrm{Cl}$ (Cambridge Isotope Laboratories) as the sole nitrogen source. The cells were grown at $18^{\circ} \mathrm{C}$ and the $\mathrm{hV1}$-S1S4 expression was induced for $48 \mathrm{~h}$ by addition of $0.1 \mathrm{mM}$ isopropyl $\beta-\mathrm{D}-1$ thiogalactopyranoside (IPTG, Research Products International Corp.) at an $\mathrm{OD}_{600 \mathrm{~nm}}$ of $0.5-0.6$. After $48 \mathrm{~h}$, the cells were harvested by centrifugation at $6000 \times g$ for $20 \mathrm{~min}$ at $4{ }^{\circ} \mathrm{C}$ resulting in a cell pellet average of ca. $2.5 \mathrm{~g}$ per liter of M9.

The cell pellet was resuspended in $25 \mathrm{~mL}$ lysis buffer $(75 \mathrm{mM}$ Tris- $\mathrm{HCl}$ (Thermo Fisher Scientific), $300 \mathrm{mM} \mathrm{NaCl}$ (Thermo Fisher Scientific), $0.5 \mathrm{mM}$ EDTA (Sigma Aldrich), pH 7.7) with $0.01 \mathrm{mg}$ of lysozyme (Sigma Aldrich), 0.01 mg DNase (Sigma Aldrich), $0.01 \mathrm{mg}$ RNase (Sigma Aldrich), 1.25 mg PMSF (Sigma Aldrich), $0.05 \mathrm{mM}$ magnesium acetate (Sigma Aldrich) and $2 \mathrm{mM} \beta$ mercaptoethanol ( $\beta$-ME, Sigma Aldrich). This lysis solution was tumbled at room temperature for $30 \mathrm{~min}$. The mixture was sonicated using an S-4000 Ultrasonic Processor (Qsonica) at $4{ }^{\circ} \mathrm{C}$ for $7.5 \mathrm{~min}$ at $55 \%$ power and $50 \%$ duty cycle of $5 \mathrm{~s}$ on and $3 \mathrm{~s}$ off. The resulting whole-cell lysate was then tumbled with $N, N$-dimethyl- $N$ dodecylglycine (Empigen BB Detergent, BOC Sciences) $(3 \% \mathrm{v} / \mathrm{v})$ for $1 \mathrm{~h}$ at $4{ }^{\circ} \mathrm{C}$ to solubilize and extract the hV1-S1S4 into micelles. After an hour, the lysate was centrifuged at $38,500 \times g$ for $30 \mathrm{~min}$ at $4{ }^{\circ} \mathrm{C}$ to pellet non-solubilized cellular debris. The supernatant was collected and tumbled with $1 \mathrm{~mL}$ of preequilibrated $\mathrm{Ni}(\mathrm{II})$ NTA Superflow (Qiagen) resin for $1-1.5 \mathrm{~h}$ at $4{ }^{\circ} \mathrm{C}$.

The hV1-S1S4-bound resin was packed in a gravity column to a column volume $\sim 1 \mathrm{~mL}$ and washed with 20 column volumes of Buffer A (40 mM HEPES (Sigma Aldrich), $300 \mathrm{mM} \mathrm{NaCl}$ (Thermo Fisher Scientific), $2 \mathrm{mM} \beta$-ME (Sigma Aldrich) $\mathrm{pH} 7.8$ ) with $1.5 \%(\mathrm{v} / \mathrm{v})$ Empigen detergent (BOC Sciences). The resin was washed with 20 column volumes of wash buffer ( $40 \mathrm{mM}$ HEPES, $300 \mathrm{mM} \mathrm{NaCl}, 60 \mathrm{mM}$ Imidazole (Sigma Aldrich), 1.5\% (v/v) Empigen, $2 \mathrm{mM} \beta-\mathrm{ME}, \mathrm{pH} 7.8$ ). With the $\mathrm{hV1} 1-\mathrm{S} 1 \mathrm{~S} 4$ bound to the resin, the detergent was exchanged into the desirable membrane mimic for further studies with 15 column volumes of rinse buffer ( 25 $\mathrm{mM} \mathrm{Na}_{2} \mathrm{HPO}_{4}$ (Thermo Fisher Scientific), 0.05\% (w/v) lyso-

palmitoylphosphatidylglycerol (LPPG, Anatrace), $2 \mathrm{mM} \beta-\mathrm{ME}, \mathrm{pH} 7.8$ ). Finally, the hV1-S1S4 was eluted with 15 column volumes of elution buffer $(25 \mathrm{mM}$ $\mathrm{Na}_{2} \mathrm{HPO}_{4}, 300 \mathrm{mM}$ Imidazole, 0.1\% (w/v) LPPG, 2 mM BME, pH 7.8).

A variety of membrane mimics were screened by ${ }^{15} \mathrm{~N}$-TROSY-HSQC NMR, including the following: DPC $(0.5 \%(\mathrm{w} / \mathrm{v}))$, TDPC $(0.5 \%(\mathrm{w} / \mathrm{v}))$, LMPC $(0.2 \%(\mathrm{w} /$ v)), LMPG (0.2\% (w/v)), LPPC $(0.2 \%(w / v))$, and LPPG $(0.2 \%(w / v))($ Fig. S1). Spectra of all tested samples were collected at both 20 and $50{ }^{\circ} \mathrm{C}$, and the spectral qualities were compared. The hV1-S1S4 purified in LPPG micelles produced the most well-resolved and dispersed spectra with the appearance of the expected number of resonances and was therefore the membrane mimic chosen for the resulting studies.

After Ni-NTA purification the hV1-S1S4 was buffer exchanged to thrombin cleavage buffer $\left(25 \mathrm{mM} \mathrm{Na}_{2} \mathrm{HPO}_{4}, 150 \mathrm{mM} \mathrm{NaCl}, \mathrm{pH}\right.$ 7.8) using an Amicon Ultra centrifugal ultrafiltration unit (Milipore, $10 \mathrm{kDa}$ cutoff). The concentration of protein was determined using a BCA kit (Thermo Fisher) and $3 \mathrm{U}$ thrombin (Novagen) was added for every $1 \mathrm{mg}$ protein. The thrombin-protein cleavage reaction was incubated at room temperature $\left(\mathrm{ca} .23^{\circ} \mathrm{C}\right)$ for $24 \mathrm{~h}$. After the reaction was completed, this mixture was passed over Ni-NTA resin with the flow through containing the thrombin-cleaved hV1-S1S4.

Gel filtration of the cleaved protein was carried out by concentrating to ca. 0.5 $\mathrm{mL}$ in NMR buffer $\left(25 \mathrm{mM} \mathrm{Na} \mathrm{HPO}_{4}, 0.5 \mathrm{mM}\right.$ EDTA, pH 6.5), and was eluted with a $1 \mathrm{CV}$ of a Superdex 200 (GE Healthcare Life Sciences) column. The fractions 
with high $A_{280}$ readings were analyzed with SDS-PAGE. The purified sample was concentrated to the desired volume. After purification, the average yield of pure hV1-S1S4 per liter of M9 medium was approximately $1.5 \mathrm{mg}$.

Validation of the hV1-S1S4 identity. To confirm the identity of purified hV1S1S4, the SDS-PAGE band was cut and sent to the MS Bioworks, which performed liquid chromatography tandem mass spectrometry (LC-MS/MS). The result revealed that our purified hV1-S1S4 matches the hV1-S1S4 amino acid sequence we provided with $51 \%$ coverage (Fig. S1b).

MTSL site directed spin labeling. For PRE maleimide chemistry was used to label the hV1-S1S4 with a nitroxide spin label MTSL (S-(1-oxyl-2,2,5,5-tetramethyl-2,5dihydro-1H-pyrrol-3-yl)methyl methanesulfonothioate, Santa Cruz Biotech). Only the WT construct containing a lone cysteine in S1 $\alpha$-helix (C443) was used for PRE. For these reactions, the hV1-S1S4 was prepared as above, except before thrombin cleavage the volume of purified hV1-S1S4 was adjusted to $0.5 \mathrm{~mL}$ in $25 \mathrm{mM}$ sodium phosphate buffer at $\mathrm{pH}$ 7.2. To maximize nitroxide labeling efficiency, purified hV1-S1S4 was incubated and tumbled with $2.5 \mathrm{mM}$ dithiothreitol (DTT, A Geno Technology, Inc.) for $1-2 \mathrm{~h}$ at room temperature to reduce the cysteine sulfhydryl. After the reaction with DTT, a 10:1 MTSL (stock solubilized in DMSO) to protein mole ratio amount of nitroxide spin label was solubilized in DMSO and incubated for $3 \mathrm{~h}$ at $37^{\circ} \mathrm{C}$. The reaction was continued at room temperature overnight and was buffer exchanged to $25 \mathrm{mM} \mathrm{Na}_{2} \mathrm{HPO}_{4}, \mathrm{pH}$ 7.8. The resulting MTSL-hV1-S1S4 was rebound to Ni-NTA resin and incubated for $1 \mathrm{~h}$ at room temperature. MTSL was removed by washing the resin with at least 20 column volumes of rinse buffer ( $25 \mathrm{mM} \mathrm{Na}_{2} \mathrm{HPO}_{4}, 0.05 \%$ (w/v) LPPG, pH 7.8). MTSL-labeled-hV1-S1S4 was eluted with five column volumes of elution buffer $\left(25 \mathrm{mM} \mathrm{Na}_{2} \mathrm{HPO}_{4}, 300 \mathrm{mM}\right.$ Imidazole, $0.1 \%(\mathrm{w} / \mathrm{v}) \mathrm{LPPG}, \mathrm{pH}$ 7.8). After this step, the purification follows as described above with thrombin cleavage and SEC.

\section{NMR resonance assignment and secondary structure analysis. All NMR} experiments were recorded with a Bruker $850 \mathrm{MHz}{ }^{1} \mathrm{H}$ spectrometer and Avance III $\mathrm{HD}$ console equipped with a $5 \mathrm{~mm}$ TCI CryoProbe. All samples included $4 \mu \mathrm{L}$ $\mathrm{D}_{2} \mathrm{O}$ with a total volume of $180 \mu \mathrm{L}$ in a $3 \mathrm{~mm}$ NMR tube. The temperature for these experiments was calibrated using $99 \%{ }^{2} \mathrm{H}$ methanol where the difference in chemical shifts (ppm) arising from the two resonances is used as a temperature standard with the ranges of $178 \mathrm{~K}$ and $330 \mathrm{~K}$ according to $T[K]=409.0-$ $36.54 \Delta \delta-21.85(\Delta \delta)^{2}$ where $\Delta \delta$ is the difference in $\mathrm{Hz}$ between the two peaks ${ }^{80}$. Experiments used for backbone resonance assignment of the hV1-S1S4 were carried out at $45{ }^{\circ} \mathrm{C}$ with a $900 \mu \mathrm{M}$ uniformly ${ }^{15} \mathrm{~N},{ }^{13} \mathrm{C}$ labeled sample in a Bruker shaped NMR tube (Part Number Z106898) with $4 \% \mathrm{D}_{2} \mathrm{O}(\mathrm{v} / \mathrm{v})$. TROSY versions of HSQC, HNCA, HNCOCA, HNCO, HNCACO, HNCACB, and CBCACONH as well as non-TROSY 4D HNCACO and 4D HNCOCA experiments were utilized, and an ${ }^{15} \mathrm{~N}$-edited HSQC-NOESY with a 90 ms mixing time $(\tau)$ was used for sequential assignments. The nonuniformly sampled data were reconstructed with qMDD and processed in nmrPipe, with analysis and resonance assignment carried out using CcpNMR ${ }^{81}$ software (Supplementary Fig. 2). The resonance assignments at $45^{\circ} \mathrm{C}$ are deposited in the Biological Magnetic Resonance Bank (BMRB entry 27029).

The secondary structure of the hV1-S1S4 at $45^{\circ} \mathrm{C}$ was determined using TALOS- $\mathrm{N}^{39}$ where the secondary structure was plotted as probability of secondary structure vs residue number. The secondary structure was then plotted in Aline $e^{82}$ alongside rTRPV1 structures determined by cryo-EM. The secondary structure for each of the rTRPV1 structures were specifically determined by analyzing the PDB codes deposited in the Protein Data Bank for requisition numbers 5IRZ, 5IRX, and 5IS0.

NMR capsaicin and temperature titrations. For the titration with capsaicin (Corden Pharma), stock capsaicin was prepared in 195 proof ethanol (Thermo Scientific), and the mole percent of capsaicin was calculated by

$$
\text { mol } \% \text { capsaicin }=\frac{(\text { moles capsaicin })}{\text { moles capsaicin }+ \text { moles LPPG }+ \text { moles hV1S1S4 }} \times 100 \% . \quad \text { (1) }
$$

Using $66 \mu \mathrm{M} \mathrm{hV1-S1S4}$ in 1.67\% LPPG $(\mathrm{w} / \mathrm{v})$, a series of ${ }^{1} \mathrm{H}-{ }^{15} \mathrm{~N}$ TROSYHSQC spectra as a function of capsaicin concentration were collected at $37^{\circ} \mathrm{C}$, with 32 transients of 2048 direct points and 128 indirect points. The hV1-S1S4 resonance assignments at $45^{\circ} \mathrm{C}$ were mapped to $37^{\circ} \mathrm{C}$ and used to identify residues that specifically interact with capsaicin (Fig. 1e). The mole\% capsaicin used were $0.001,0.002,0.005,0.012,0.015,0.025,0.075,0.125,0.225$ and $0.723 \%$ (Fig. 1d). These spectra were processed with nmrPipe ${ }^{83}$ and the resonances were analyzed in $\mathrm{CcpNmr}{ }^{81}$. Chemical shift perturbations induced by capsaicin were calculated by

$$
\Delta \delta=\sqrt{\left(\Delta \delta_{\mathrm{H}}\right)^{2}+\left(0.2\left(\Delta \delta_{\mathrm{N}}\right)\right)^{2}}
$$

where $\Delta \delta_{\mathrm{H}}$ is the difference in chemical shift in the proton dimension and $\Delta \delta_{\mathrm{N}}$ is the difference in chemical shift in the nitrogen dimension, and the $\Delta \delta_{\mathrm{N}}$ was multiplied by a scaling factor of 0.2 typically used for nitrogen nuclei ${ }^{84}$. The capsaicin-dependent chemical shift perturbations were plotted as a function of the mole $\%$ of added capsaicin and fit to a single site binding model derived from the
Langmuir adsorption model commonly used to describe binding in proteins:

$$
\Delta \delta(\text { mole } \%)=\frac{\Delta \delta_{\max } \times \text { mole } \%}{K_{\mathrm{d}}+\text { mole } \%},
$$

where $K_{\mathrm{d}}$ is the dissociation constant and $\Delta \delta_{\max }$ is the maximal value of change in chemical shift from perturbation. Using the amide backbone assignment of the hV1-S1S4, the chemical shift perturbations of 72 residues were analyzed and the $K_{\mathrm{d}}$ values for each residue were calculated using MATLAB R2016afit to equation [3].

NMR temperature tirations used ${ }^{1} \mathrm{H}_{-}{ }^{15} \mathrm{~N}$ TROSY-HSQC experiments of the purified hV1-S1S4 and were recorded from 15 to $50^{\circ} \mathrm{C}$, at $5^{\circ} \mathrm{C}$ increments with all other parameters fixed. Specific types of buffers exhibit $\mathrm{pH}$ changes at different temperatures, an effect that could convolute our analyses. To ensure that the chemical shift perturbations were caused exclusively by temperature changes and not from $\mathrm{pH}$ changes of the buffer, ${ }^{1} \mathrm{H}_{-}{ }^{15} \mathrm{~N}$-TROSY-HSQC spectra at two different $\mathrm{pH}$ values, 6.5 and 6.4, were collected at $37^{\circ} \mathrm{C}$ (Supplementary Fig. 5). These two $\mathrm{pH}$ values were chosen based on empirical $\mathrm{pH}$ changes measured with our NMR buffer as a function of temperature (Supplementary Table 2). No chemical shift perturbations were detected allowing direct analysis for their dependence on temperature-dependent resonance changes.

The resonance intensities (peak height) of several hV1-S1S4 residues were plotted against the temperature, and fit to a sigmoidal two-state model using $M A T L A B$ scripts relying on the nlinfit function with the following equation derived from a Van't Hoff model:

$$
I(T)=\frac{\left(I_{\text {active }}+I_{\text {resting }}\right) \mathrm{e}^{\left(-\frac{\Delta \mathrm{H}}{R}\left(\frac{1}{T}-\frac{1}{T_{m}}\right)\right)}}{1+\mathrm{e}^{\left(-\frac{\Delta \mathrm{H}}{R}\left(\frac{1}{T}-\frac{1}{T_{m}}\right)\right)}} .
$$

From this equation, the changes in enthalpies $(\Delta \mathrm{H})$ of 71 residues for WT and 70 residues for the hV1-S1S4-R557A mutant residues were obtained. For the WT, three distinct samples were used for the experiments, yielding three sets of the $\Delta \mathrm{H}$ values per residue. From the enthalpies, a histogram with bins of $5 \mathrm{kcal} \mathrm{mol}^{-1}$ was fit to a Gaussian function, $f(\Delta \mathrm{H})=a_{\max } \exp \left(-\frac{(\Delta \mathrm{H}-\mu)^{2}}{2 \sigma^{2}}\right)$, in MATLAB for both WT and R557A, where $\mu$ and $\sigma$ are the ensemble average enthalpy and variance respectively. To help validate the experimental NMR-detected temperature titration data, simulations of NMR peak height (resonance intensity) under conditions that mirror the experimental conditions were carried out. These simulations are detailed further in the Supplementary Methods and shown in Supplementary Fig. 7 with numerical parameters listed in Supplementary Table 5.

NMR TROSY for rotational correlation times measurements. To accurately quantify the PRE-derived distances between individual indole amine (W549 and W427) residues and the MTSL-labeled C443, accurate rotational correlation times $\left(\tau_{c}\right)$ are needed at $20^{\circ} \mathrm{C}$ and $50^{\circ} \mathrm{C}$. To approximate $\tau_{c}$, TRACT ${ }^{51}$ was collected over a range of time delays from $\tau=0$ to $100 \mathrm{~ms}$ with $4 \mathrm{~ms}$ intervals. The pulse program that was used for these experiments was adapted from Lee et al ${ }^{51}$ The data were processed and integrated in nmrPipe over a range of 9.6 to $10 \mathrm{ppm}$, to which a linear baseline correction was applied with the nmrPipe function BASE and a defined node list in the noise (Supplementary Fig. 6). The range that was integrated was selected as it encompassed the W549 indole amine resonance from the S4 transmembrane helix, and only this was integrated since integration over the entire amino region can artificially inflate the effective correlation time ${ }^{85}$. The integrated data was fit using MATLAB R2015a with a monoexponential decay function, $f(\tau)=A \mathrm{e}^{-\beta \tau}$, where $A$ is a normalized maximum, $\tau$ is the time delay, and $\beta$ is the relaxation decay constant in $\mathrm{Hz}$. The relaxation decays for the $\alpha$ and $\beta$ state of exclusively the W549 indole amine resonance were used to calculate the approximate effective rotational correlation time $\left(\tau_{c}\right)$ due to the rigid body assumption as described previously by Lee et al. ${ }^{51}$

Calculation of amide proton temperature coefficients. From the NMR temperature titration from 15 to $50^{\circ} \mathrm{C}(288-323 \mathrm{~K})$, the amide proton chemical shifts $\left(\delta_{\mathrm{HN}}\right)$ of assigned resonances at corresponding temperatures were plotted in the same temperature range. Each plot was fit to the linear function, $y=m x+b$, where the slope is the temperature coefficient, $\Delta \delta_{\mathrm{HN}} / \Delta T\left(\mathrm{ppb} \mathrm{K}^{-1}\right)$.

Amide proton resonances are predominantly influenced by their hydrogenbonded state, and the temperature-dependent changes are predominantly linear, reflecting protein thermal expansion and a corresponding increase in hydrogen bond length ${ }^{50,86,87}$. Small non-linear deviations of the temperature dependence of amide proton chemical shifts are sometimes detected ${ }^{49,88}$. These curved profiles result from accessible conformational exchange between ground and excited protein states ${ }^{50}$. To date quantification of temperature coefficient non-linearity has occurred from fitting the residuals of the linear fit to a quadratic equation where the sign and magnitude of the first quadratic coefficient is a metric for dynamics. However, the quadratic nature of the residuals is empirical in nature, and there is currently no theoretical reason to expect a quadratic relationship from the data ${ }^{50}$ Given that our NMR, CD, and fluorescence temperature titrations indicate that the hV1-S1S4 domain undergoes a transition between conformational states between lower and elevated temperatures, we posit that the residuals from the linear temperature coefficient would be non-linear. However, given that the aforementioned data suggest that the hV1-S1S4 changes conformation, as opposed 
to transiently accessing an alternate conformational state, we anticipate that this would be reflected in analysis of the residuals. Moreover, because the "ground" state changes between low and elevated temperatures, it is expected that the residual fits would deviate from quadratic. Indeed, analysis of the residual chemical shift from the hV1-S1S4 amide proton chemical shift data show a higher order polynomial relationship that fits well to a cubic equation which would be the form if there was a slight sigmoidal shape superimposed on the linear expansion (Supplementary Fig. 8). Taken together, residual chemical shift analysis of temperature coefficients support the NMR peak height, $\mathrm{CD}$, and intrinsic tryptophan fluorescence conclusions that there is a conformational change in hV1SiS4 effected from temperature change. To our knowledge, a similar non-zero/ non-quadratic residual profile has not been previously reported. We interpret this as supporting the hV1-S1S4 temperature-dependent conformational states identified with other experiments.

NMR PRE measurements. Paramagnetic (MTSL labeled) and diamagnetic (without the MTSL label) proton transverse relaxation $\left(R_{2}\right)$ measurements were recorded using a TROSY-HSQC pulse program modified to include a relaxation delay before acquisition as described by Clore and coworkers ${ }^{89}$. Four relaxation delays $(0,4,12,24 \mathrm{~ms})$ were recorded for the matched paramagnetic and diamagnetic samples at $20^{\circ} \mathrm{C}$ (196 scans) and $50{ }^{\circ} \mathrm{C}$ (80 scans) with 2048 direct point and 128 indirect points. The spectra were processed using nmrPipe with a Gaussian apodization function, which does not affect the calculated distance to the free electron $\operatorname{spin}^{90}$. The resonance intensity (peak height) values were obtained using $\mathrm{CcpNmr}$ and transverse relaxation rates of the paramagnetic state, $R_{2}{ }^{\text {eff }}$, and the diamagnetic state, $R_{2}$, were extracted by fitting the data with a monoexponential decay function, $y=a \mathrm{e}^{-b x}$ (Supplementary Fig. 6). The paramagnetic rate enhancements, $\Gamma_{2}$, were calculated by subtracting $R_{2}$ from $R_{2}{ }_{2}^{\text {eff }}$, using the relationship of the electron spin-enhanced transverse relaxation rate due to the spin label $\left(R_{2}{ }^{\text {eff }}\right)$ is a sum of the intrinsic transverse relaxation rate $\left(R_{2}\right)$ and the contribution from the electron $\left(\Gamma_{2}\right)$ :

$$
R_{2}^{\text {eff }}=\Gamma_{2}-R_{2}
$$

The errors for $R_{2}{ }^{\text {eff }}$ and $R_{2}$ were extracted from fitting errors, and the error of $\delta \Gamma_{2}$ was calculated using standard the propagation of error:

$$
\delta \Gamma_{2}=\sqrt{\left(\delta R_{2}^{\text {eff }}\right)^{2}+\left(\delta R_{2}\right)^{2}} .
$$

The distance restraints from MTSL-labeled C443 to residues in proximity were calculated by the following relationship:

$$
r=\left[\frac{K}{\Gamma_{2}}\left(4 \tau_{\mathrm{c}}+\frac{3 \tau_{\mathrm{c}}}{1+\omega_{\mathrm{H}}^{2} \tau_{\mathrm{c}}^{2}}\right)\right]^{\frac{1}{6}},
$$

where $\tau_{\mathrm{c}}$ is the effective rotational correlation time, $\omega_{\mathrm{H}}$ is the Larmor frequency for the nuclear spin (protons), and $K$ is $1.23 \times 10^{-32} \mathrm{~cm}^{6} \mathrm{~s}^{-2}$ a constant related to the gyromagnetic ratio $(\gamma)$, spin quantum number $(S)$, electronic $\mathrm{g}$ factor $(g)$, and the Bohr magneton $(\beta)$ according to $K=\frac{1}{15} S(S+1) \gamma^{2} g^{2} \beta^{2}$.

Three separate paramagnetic (MTSL labeled) and diamagnetic (non-MTSL labeled) samples were prepared. Transverse relaxation rates of states were obtained, and using various combinations, 9 paramagnetic enhancement rates were calculated, generating nine distance restraints per residue. Distances calculated to be higher than $27 \AA$ were disregarded as this is beyond the reliable distance calculation for the atoms involved due to its $\mathrm{r}^{-6}$ dependence. The distances at $20^{\circ} \mathrm{C}$ and $50^{\circ} \mathrm{C}$ were averaged, and the temperature-dependent change in distances ( $\triangle \mathrm{PRE}$ ) were calculated by subtracting $20^{\circ} \mathrm{C}$ (resting state, initial) from $50^{\circ} \mathrm{C}$ (active state, final) $\left(\triangle \mathrm{PRE}_{50}{ }^{\circ} \mathrm{C}-20^{\circ} \mathrm{C}\right)$. We interpreted that the residues with negative $\triangle$ PREs are moving closer to MTSL-C443, and the residues with positive $\triangle$ PREs are moving away from MTSL-C443. The errors of distance restraints were the standard error of the mean (s.e.m.).

NMR NOESY experiments at two different temperatures. ${ }^{15} \mathrm{~N}$-edited TROSYHSQC-NOESY spectra of the hV1-S1S4 were collected with 96 scans and 48 scans with the mixing time of $90 \mathrm{~ms}$ at $20^{\circ} \mathrm{C}$ and $45^{\circ} \mathrm{C}$, respectively. Using the amide resonance assignments and temperature titration resonances, amide NOESY crosspeaks were assigned to corresponding $\mathrm{Ha}$ and water cross-peaks. Using peak volumes, the ratio $(I)$ between the water and $\mathrm{H} \alpha$ cross-peaks were calculated by dividing the water cross-peak by $\mathrm{Ha}$ cross-peak at both temperatures. Then the differences of $I_{\text {water }}: I_{\mathrm{H \alpha}}$ at $45^{\circ} \mathrm{C}$ and $20^{\circ} \mathrm{C}\left(\Delta \mathrm{NOE}_{45}{ }^{\circ} \mathrm{C}-20^{\circ} \mathrm{C}\right)$ were calculated for all well-resolved resonances to observe if the hV1-S1S4 undergoes changes in hydration as a function of temperature. The $\triangle \mathrm{NOE}_{45}{ }^{\circ} \mathrm{C}-20^{\circ} \mathrm{C}$ was plotted against the residue number and the cutoff value of 0.5 was used to evaluate the changes in hydration per residue.

NMR deuterium/hydrogen exchange factor analysis. The $\mathrm{H}_{2} \mathrm{O}: \mathrm{D}_{2} \mathrm{O}$ exchange measurement for the hV1-S1S4 was carried out at $50{ }^{\circ} \mathrm{C}$. To vary the concentration of $\mathrm{D}_{2} \mathrm{O}$ in the sample, the NMR buffer was prepared in $\mathrm{D}_{2} \mathrm{O}$. This was done by lyophilizing $500 \mu \mathrm{L}$ of NMR buffer and resuspending it in $500 \mu \mathrm{L}$ of $\mathrm{D}_{2} \mathrm{O}$. To make $10 \% \mathrm{D}_{2} \mathrm{O}: 90 \% \mathrm{H}_{2} \mathrm{O}, 18 \mathrm{uL}$ of NMR buffer in $\mathrm{D}_{2} \mathrm{O}$ was added to the sample, and the sample was incubated for approximately $12 \mathrm{~h}$ to ensure that the exchange between the proton and deuterium is completed. ${ }^{1} \mathrm{H}^{-15} \mathrm{~N}$ TROSY-HSQC was collected as described above with 32 scans. For the higher concentration of $\mathrm{D}_{2} \mathrm{O}$ titration points, the sample with $10 \% \mathrm{D}_{2} \mathrm{O}: 90 \% \mathrm{H}_{2} \mathrm{O}$ was concentrated down to approximately $100 \mu \mathrm{L}$, and $80 \mu \mathrm{L}$ of the NMR buffer in $\mathrm{D}_{2} \mathrm{O}$ was added to the concentrated sample to make a $40 \% \mathrm{D}_{2} \mathrm{O}: 60 \% \mathrm{H}_{2} \mathrm{O}$ sample. For the further $\mathrm{D}_{2} \mathrm{O}$ points, the same method was used.

The resulting data were analyzed with a method developed by Opella et al. ${ }^{91}$. The relative peak volumes of resonances were normalized to those from the $2.2 \%$ $\mathrm{D}_{2} \mathrm{O}$ spectrum. The mole fraction of water $\left(\chi_{\text {water }}\right)$ was calculated for each data point and plotted against the normalized peak volumes. The decay in resonance intensity as a function of $\chi_{\text {water }}$ is linearly dependent on the exchange factor $m$. All $\mathrm{D} / \mathrm{H}$ assigned exchange factors were plotted against the residue number for further analysis.

NMR residual dipolar coupling (RDC). A neutral 3.8\% polyacrylamide gel comprising 3.8\% (w/v) copolymer was generated using acrylamide (stock $40 \% \mathrm{w} / \mathrm{v}$, Sigma-Aldrich), bis-acrylamide (stock $2 \%$, Sigma-Aldrich), $10 \%$ APS, and $4 \mu \mathrm{L}$ of TEMED in a casting solution of $10 \mathrm{~mL}$ of $250 \mathrm{mM}$ imidazole at $\mathrm{pH}$ 6.5. These gels were cast in a 3D printed plastic block with a custom $6 \mathrm{~mm}$ inner diameter polytetrafluoroethylene (PTFE, Teflon) tube insertion. Prior to adding TEMED, the solution was filtered using $0.22 \mu \mathrm{m}$ filter (Millipore) to eliminate any polymerized acrylamide. The polymerization reaction was carried out for $24 \mathrm{~h}$. Once polymerization was complete, the gel was soaked in $\mathrm{ddH}_{2} \mathrm{O}$ for $\sim 12 \mathrm{~h}$ initially, then it was dialyzed in the NMR buffer for $48 \mathrm{~h}$ with a gentle nutating at room temperature; with buffer changes every $12-18 \mathrm{~h}$. Once the dialysis was complete, the gel was cut to $1.7 \mathrm{~mm}$ length; the length was empirically chosen to span the probe coil within a $5 \mathrm{~mm}$ NMR tube. The cut gel was partially dehydrated by incubating at $37^{\circ} \mathrm{C}$ for $3.5 \mathrm{~h}$, which was then soaked in $1 \mathrm{~mL}$ of a purified $177 \mu \mathrm{M} \mathrm{hV1-S1S4}$ sample for approximately $48 \mathrm{~h}$. The concentration of the protein solution outside of the gel was measured every $24 \mathrm{~h}$ to monitor absorption into the gel. Once concentration plateaued, the protein-soaked gel was stretched into a $5 \mathrm{~mm}$ NMR tube with an inner diameter of $4.4 \mathrm{~mm}$ using Tygon tubing attached to the NMR tube and a syringe, the difference in gel and tube diameter stretched the gel to $\sim 4 \mathrm{~mm}$. Residual dipolar couplings were measured using Amide RDC's by TROSY Spectroscopy (ARTSY) by taking the ratio of assigned amino acid intensities from attenuated spectra and reference spectra ${ }^{92}$. The dipolar couplings were then plotted as a function of residue number and fit according to the following equation:

$$
\delta_{\mathrm{NH}}=A \cdot \sin \left[\frac{2 \pi}{T}\left(x-x_{0}\right)+\varphi\right]+A_{\text {mean }},
$$

where $T$ is the period of the wave, $x$ is the amino acid identity, $x_{0}$ is the first amino acid to be fit in a given range, $A_{\text {mean }}$ is the average of the couplings in a given range, and $A$ represents a function of the alignment tensor according to

$$
A=\frac{2 D_{a} \cos ^{2}[\delta] \cos ^{2}[\theta]-\cos ^{2} D_{a} \cos ^{2}[\delta] \sin ^{2}[\theta]+\frac{3}{2} D_{a} R \cos ^{2}[\delta] \sin ^{2}[\theta] \cos ^{2}[\phi]}{\sin [\varphi]},
$$

where $R$ and $D_{a}$ are the rhombicity and axial symmetry of the alignment tensor, respectively. The angles $\theta$ and $\phi$ correspond to the amide bond vector angles with respect to the $Y$ - and $Z$ - axes, and $\delta$ the angle that the amide bond vector makes with the chain axis ${ }^{93}$. The first equation is a good approximation for ideal $\alpha$-helices and $3_{10}$-helices as previously described ${ }^{93,94}$ RMSE was calculated according to

$$
\text { RMSE }=\sqrt{\frac{\sum_{t=1}^{T}\left(\hat{y}_{t}-y_{t}\right)^{2}}{T}},
$$

where $\hat{y}_{t}$ corresponds to predicted values of $y_{t}$ over $T$ observations.

Comparison of the per-residue experimental RDC values with values predicted from rTRPV1 and mTRPV 3 cryo-EM structures were carried out with the software PALES (prediction of alignment from structure) using established protocols ${ }^{44,95}$. Specifically, the three $\overline{\mathrm{rT}} \mathrm{RPV} \overline{1}$ structures used to predict RDC values include the apo-, antagonist-, and agonist-bound states (PDB 5irz, 5is0, and 5irx, respectively). Additionally, RDC values were predicted from two mTRPV3 structures, one in a closed state at $42^{\circ} \mathrm{C}$ and the second of a mutant in the open state at $37^{\circ} \mathrm{C}(\mathrm{PDB}$ 6 pvl and 6pvp respectively). Only the transmembrane domain experimental RDC values were used in analysis. Amide hydrogen atoms were added to the cryo-EM structures with the Reduce software ${ }^{96}$.

Far-ultraviolet circular dichroism (Far-UV CD). Far-UV CD measurements were carried out on a $0.2 \mathrm{mg} \mathrm{ml}^{-1} \mathrm{hV} 1-\mathrm{S} 1 \mathrm{~S} 4$ sample in $0.1 \%$ (w/v) LPPG micelles in 200 $\mu \mathrm{L}$ in $25 \mathrm{mM} \mathrm{Na}_{2} \mathrm{PO}_{4}$ buffer at $\mathrm{pH}$ 6.5. CD spectra were obtained with a Jasco J-710 spectropolarimeter using a $1.0 \mathrm{~mm}$ path length cell. The temperature was controlled with a Peltier device (Jasco PTC-424S). All experiments were measured from $250 \mathrm{~nm}$ to $190 \mathrm{~nm}$ and 5 scans from $10^{\circ} \mathrm{C}$ to $57^{\circ} \mathrm{C}$ in $1^{\circ} \mathrm{C}$ increments. The blank (25 $\mathrm{mM} \mathrm{Na}_{2} \mathrm{HPO}_{4}, \mathrm{pH} 6.5$, containing $0.1 \%$ (w/v) LPPG) measurement values were subtracted from the protein measurement values, and the units of $\mathrm{CD}$ values were converted into mean residue ellipticity using the equation:

$$
[\theta]_{\mathrm{MR}}=100 \times \frac{\theta}{C \times N \times l},
$$

where $[\theta]_{\mathrm{MR}}$ is the mean residue ellipticity, $\theta$ is the ellipticity in millidegrees, $C$ is 
the concentration of protein in molarity $(\mathrm{M}), N$ is the number of amino acid residues of protein, and $l$ is the path length in centimeters. The hV1-S1S4 CD values at $222 \mathrm{~nm}$ were plotted against temperature (Fig. $2 \mathrm{~b}$ ).

\section{Temperature-dependent intrinsic tryptophan fluorescence. Fluorescence} emission spectra were measured on a QM-4/2005SE Spectrofluorometer (PTI, NJ) using $295 \mathrm{~nm}$ excitation to minimize contributions from tyrosine residues ${ }^{38}$. The temperature was controlled over a range of ca. $7^{\circ} \mathrm{C}-50^{\circ} \mathrm{C}$ using a water circulation system and a calibrated thermocouple was used to measure the temperature of the sample inside the cuvette. To account for light scattering contributions, a sample of LPPG micelles and buffer was used as a blank and subtracted from the measurements containing hV1-S1S4. The blank signals are relatively small and their magnitude and position do not change with temperature (Supplementary Fig. 5). In contrast, for the hV1-S1S4 samples, increasing temperature resulted in both a decrease in fluorescence intensity and a spectral shift to higher wavelengths. We characterize fluorescence spectral shifts in terms of the average emission wavelength, $\langle\lambda\rangle$ :

$$
\lambda=\frac{\sum I\left(\lambda_{i}\right) \lambda_{i}}{\sum I\left(\lambda_{i}\right)}
$$

where $I(\lambda)_{i}$ is the intensity measured at wavelength $\lambda_{i}$. This quantity is more precise and less sensitive to instrumental noise than the peak maximum $\left(\lambda_{\max }\right)$ because the calculation reflects data from the entire spectrum rather than a single point ${ }^{97}$.

Whole-cell patch-clamp electrophysiology experiments. Cell culture of HEK293 cells (ATCC cell line CRL-1573) used $35 \mathrm{~mm}$ dishes at $37^{\circ} \mathrm{C}$ in DMEM with

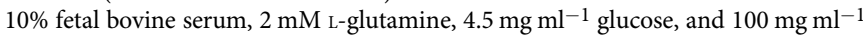
each of penicillin and streptomycin in the presence of $5 \% \mathrm{CO}_{2}$. All reagents were obtained from Life Technologies.

The full-length human TRPV1 gene was subcloned into a pIRES-2 plasmid also containing the EGFP gene. This construct produces bicistronic mRNA containing an internal ribosome entry site (IRES) between the two genes, allowing for the independent translation of TRPV1 and the EGFP reporter. TRPV1-Arg557Ala was generated in the same plasmid using standard site-directed mutagenesis. Cells were transiently transfected with $0.4 \mu \mathrm{g}$ DNA using FuGENE 6 transfection reagent (Promega) in a 1:3 $\mu \mathrm{g}$ DNA: $\mu \mathrm{L}$ FuGENE ratio $48 \mathrm{~h}$ before electrophysiology measurements were performed.

Transfected cells were released from the culture dish surface by briefly exposing them to $0.25 \%$ trypsin-EDTA (Thermo) and resuspending in supplemented DMEM. Cells were plated on glass coverslips and incubated for $1-2 \mathrm{~h}$ at $37^{\circ} \mathrm{C}$. Whole-cell voltage-clamp measurements were performed with an Axopatch 200B amplifier and pClamp 10.3 software (Molecular Devices). Data was acquired at $2 \mathrm{kHz}$, filtered at $1 \mathrm{kHz}$, and digitized using a Digidata $1440 \mathrm{a}$ digitizer (Molecular Devices). Patch pipettes were pulled from borosilicate glass capillaries (World Precision Instruments) using a P-2000 laser puller (Sutter Instruments) and heat polished with a MF-830 microforge (Narashige), resulting in resistances of 2-5 M $\Omega$. A reference electrode was inserted into a salt bridge composed of $2 \%$ agar in extracellular solution. Glass coverslips plated with cells were placed in a chamber and covered with extracellular solution containing 132 $\mathrm{mM} \mathrm{NaCl}, 5 \mathrm{mM} \mathrm{KCl}, 1 \mathrm{mM} \mathrm{MgCl} 2,2 \mathrm{mM} \mathrm{CaCl}_{2}, 10 \mathrm{mM}$ HEPES, and $5 \mathrm{mM}$ glucose. The $\mathrm{pH}$ of the solution was adjusted to 7.4 using $\mathrm{NaOH}$ and osmolality was adjusted to $310 \mathrm{mOsm}$ using sucrose. Pipette solution contained $135 \mathrm{mM} \mathrm{K}^{+}$ gluconate, $5 \mathrm{mM} \mathrm{KCl}, 1 \mathrm{mM} \mathrm{MgCl} 2,5 \mathrm{mM}$ EGTA, and $10 \mathrm{mM} \mathrm{HEPES}$, with $\mathrm{pH}$ adjusted to 7.2 using $\mathrm{KOH}$ and osmolality adjusted to $300 \mathrm{mOsm}$ using sucrose. Osmolality was measured using a Vapro 5600 vapor pressure osmometer (Wescor). Temperature was controlled by perfusing preheated or cooled extracellular solution using an HCPC perfusion system and HCT-10 temperature controller (ALA Scientific), which heats or cools solution by supplying a specified voltage to a Peltier device through which perfusion solution flows. Temperature was calibrated by measuring the temperature of the solution exiting the $\mathrm{HCPC}$ at a given voltage. Currents were recorded at $+60 \mathrm{mV}$ and normalized to cell membrane capacitance. The temperature ramp electrophysiology data were fit to a simplified Boltzmann exponential function, $P_{o}=e^{-\frac{\Delta H}{R T}+\frac{\Delta S}{R}}$, assuming a pseudo-steady state approximation to estimate the thermosensitivity $(\Delta \mathrm{H}) . \mathrm{pH}$ series measurements were carried out on individual cells subjected to a range of $\mathrm{pH}$ values from $\mathrm{pH} 7.4$ to $\mathrm{pH} 5.0$ with current evaluated at $+100 \mathrm{mV}$. Errors in the $\mathrm{pH}$ buffers are estimated to be $<$ \pm 0.05 .

Reporting summary. Further information on research design is available in the Nature Research Reporting Summary linked to this article.

\section{Data availability}

Data supporting the findings of this manuscript are available from the corresponding author upon reasonable request. A reporting summary for this Article is available as a Supplementary Information file.

Source data are provided with this paper.
The NMR resonance assignments have been deposited to the BMRB, ID: 27029. Source data are provided with this paper.

Received: 16 January 2020; Accepted: 30 July 2020; Published online: 20 August 2020

\section{References}

1. Hilton, J. K., Rath, P., Helsell, C. V., Beckstein, O. \& Van Horn, W. D. Understanding thermosensitive transient receptor potential channels as versatile polymodal cellular sensors. Biochemistry 54, 2401-2413 (2015)

2. Voets, T. TRP channels and thermosensation. Handb. Exp. Pharmacol. 223, 729-741 (2014).

3. Caterina, M. J. et al. The capsaicin receptor: a heat-activated ion channel in the pain pathway. Nature 389, 816-824 (1997).

4. Kim, A. Y. et al. Pirt, a phosphoinositide-binding protein, functions as a regulatory subunit of TRPV1. Cell 133, 475-485 (2008)

5. Klein, R. M., Ufret-Vincenty, C. A., Hua, L. \& Gordon, S. E. Determinants of molecular specificity in phosphoinositide regulation. Phosphatidylinositol $(4,5)$-bisphosphate $(\mathrm{PI}(4,5) \mathrm{P} 2)$ is the endogenous lipid regulating TRPV1. J. Biol. Chem. 283, 26208-26216 (2008).

6. Tominaga, M. et al. The cloned capsaicin receptor integrates multiple painproducing stimuli. Neuron 21, 531-543 (1998).

7. Fernandes, E. S., Fernandes, M. A. \& Keeble, J. E. The functions of TRPA1 and TRPV1: moving away from sensory nerves. Br. J. Pharmacol. 166, 510-521 (2012)

8. Julius, D. TRP channels and pain. Annu. Rev. Cell. Dev. Biol. 29, 355-384 (2013).

9. Moran, M. M., McAlexander, M. A., Biro, T. \& Szallasi, A. Transient receptor potential channels as therapeutic targets. Nat. Rev. Drug Discov. 10, 601-620 (2011).

10. Fernandes, E. S. et al. TRPV1 deletion enhances local inflammation and accelerates the onset of systemic inflammatory response syndrome. $J$. Immunol. 188, 5741-5751 (2012).

11. Razavi, R. et al. TRPV1 sensory neurons control beta cell stress and islet inflammation in autoimmune diabetes. Cell 127, 1123-1135 (2006).

12. Lee, L. Y. \& Gu, Q. Role of TRPV1 in inflammation-induced airway hypersensitivity. Curr. Opin. Pharmacol. 9, 243-249 (2009).

13. Motter, A. L. \& Ahern, G. P. TRPV1-null mice are protected from dietinduced obesity. FEBS Lett. 582, 2257-2262 (2008).

14. Suri, A. \& Szallasi, A. The emerging role of TRPV1 in diabetes and obesity. Trends Pharmacol. Sci. 29, 29-36 (2008).

15. Riera, C. E. et al. TRPV1 pain receptors regulate longevity and metabolism by neuropeptide signaling. Cell 157, 1023-1036 (2014).

16. Mistretta, F. et al. Bladder cancer and urothelial impairment: the role of TRPV1 as potential drug target. Biomed. Res. Int. 2014, 987149 (2014).

17. Stock, K. et al. Neural precursor cells induce cell death of high-grade astrocytomas through stimulation of TRPV1. Nat. Med. 18, 1232-1238 (2012).

18. Hilton, J. K., Kim, M. \& Van Horn, W. D. Structural and Evolutionary Insights Point to Allosteric Regulation of TRP Ion Channels. Acc. Chem. Res. 52, 1643-1652 (2019).

19. Liao, M., Cao, E., Julius, D. \& Cheng, Y. Structure of the TRPV1 ion channel determined by electron cryo-microscopy. Nature 504, 107-112 (2013).

20. Cao, E., Liao, M., Cheng, Y. \& Julius, D. TRPV1 structures in distinct conformations reveal activation mechanisms. Nature 504, 113-118 (2013).

21. Gao, Y., Cao, E., Julius, D. \& Cheng, Y. TRPV1 structures in nanodiscs reveal mechanisms of ligand and lipid action. Nature 534, 347-351 (2016).

22. Yang, F. et al. The conformational wave in capsaicin activation of transient receptor potential vanilloid 1 ion channel. Nat. Commun. 9, 2879 (2018).

23. Jordt, S.-E. \& Julius, D. Molecular basis for species-specific sensitivity to "hot" chili peppers. Cell 108, 421-430 (2002).

24. Gavva, N. R. et al. Molecular determinants of vanilloid sensitivity in TRPV1. J. Biol. Chem. 279, 20283-20295 (2004).

25. Sutton, K. G. et al. Functional characterisation of the S512Y mutant vanilloid human TRPV1 receptor. Br. J. Pharmacol. 146, 702-711 (2005).

26. Yang, F. et al. Structural mechanism underlying capsaicin binding and activation of the TRPV1 ion channel. Nat. Chem. Biol. 11, 518-524 (2015).

27. Hanson, S. M., Newstead, S., Swartz, K. J. \& Sansom, M. S. Capsaicin interaction with TRPV1 channels in a lipid bilayer: molecular dynamics simulation. Biophys. J. 108, 1425-1434 (2015).

28. Elokely, K. et al. Understanding TRPV1 activation by ligands: Insights from the binding modes of capsaicin and resiniferatoxin. Proc. Natl Acad. Sci. U. S A. 113, E137-E145 (2016).

29. Clapham, D. E. \& Miller, C. A thermodynamic framework for understanding temperature sensing by transient receptor potential (TRP) channels. Proc. Nat Acad. Sci. USA 108, 19492-19497 (2011). 
30. Liu, B., Hui, K. \& Qin, F. Thermodynamics of heat activation of single capsaicin ion channels VR1. Biophys. J. 85, 2988-3006 (2003).

31. Yao, J., Liu, B. \& Qin, F. Kinetic and energetic analysis of thermally activated TRPV1 channels. Biophys. J. 99, 1743-1753 (2010).

32. Chowdhury, S., Jarecki, B. W. \& Chanda, B. A molecular framework for temperature-dependent gating of ion channels. Cell 158, 1148-1158 (2014).

33. Lolicato, M., Riegelhaupt, P. M., Arrigoni, C., Clark, K. A. \& Minor, D. L. Jr. Transmembrane helix straightening and buckling underlies activation of mechanosensitive and thermosensitive K(2P) channels. Neuron 84, 1198-1212 (2014).

34. Cao, E., Cordero-Morales, J. F., Liu, B., Qin, F. \& Julius, D. TRPV1 channels are intrinsically heat sensitive and negatively regulated by phosphoinositide lipids. Neuron 77, 667-679 (2013).

35. Zaelzer, C. et al. DeltaN-TRPV1: a molecular co-detector of body temperature and osmotic stress. Cell. Rep. 13, 23-30 (2015).

36. Zhang, F. et al. Heat activation is intrinsic to the pore domain of TRPV1. Proc. Natl Acad. Sci. U. S. A. 115, E317-E324 (2018).

37. Greenfield, N. J. Using circular dichroism collected as a function of temperature to determine the thermodynamics of protein unfolding and binding interactions. Nat. Protoc. 1, 2527-2535 (2006).

38. Royer, C. A. Probing protein folding and conformational transitions with fluorescence. Chem. Rev. 106, 1769-1784 (2006).

39. Shen, Y. \& Bax, A. Protein structural information derived from NMR chemical shift with the neural network program TALOS-N. Methods Mol. Biol. 1260, 17-32 (2015).

40. Asakura, T., Taoka, K., Demura, M. \& Williamson, M. P. The relationship between amide proton chemical shifts and secondary structure in proteins. $J$. Biomol. NMR 6, 227-236 (1995).

41. Zhou, N. E., Zhu, B. Y., Sykes, B. D. \& Hodges, R. S. Relationship between amide proton chemical shifts and hydrogen bonding in amphipathic.alpha. helical peptides. J. Am. Chem. Soc. 114, 4320-4326 (1992).

42. Shenkarev, Z. O. et al. NMR structural and dynamical investigation of the isolated voltage-sensing domain of the potassium channel KvAP: implications for voltage gating. J. Am. Chem. Soc. 132, 5630-5637 (2010).

43. Mesleh, M. F. et al. Dipolar waves map the structure and topology of helices in membrane proteins. J. Am. Chem. Soc. 125, 8928-8935 (2003).

44. Zweckstetter, M. \& Bax, A. Evaluation of uncertainty in alignment tensors obtained from dipolar couplings. J. Biomol. NMR 23, 127-137 (2002).

45. Chen, K. \& Tjandra, N. The use of residual dipolar coupling in studying proteins by NMR. Top. Curr. Chem. 326, 47-67 (2012).

46. Kabsch, W. \& Sander, C. Dictionary of protein secondary structure: pattern recognition of hydrogen-bonded and geometrical features. Biopolymers 22, 2577-2637 (1983).

47. Touw, W. G. et al. A series of PDB-related databanks for everyday needs. Nucleic Acids Res. 43, D364-D368 (2015).

48. Baxter, N. J. \& Williamson, M. P. Temperature dependence of $1 \mathrm{H}$ chemical shifts in proteins. J. Biomol. NMR 9, 359-369 (1997).

49. Doyle, C. M. et al. Concurrent increases and decreases in local stability and conformational heterogeneity in $\mathrm{Cu}, \mathrm{Zn}$ superoxide dismutase variants revealed by temperature-dependence of amide chemical shifts. Biochemistry 55, 1346-1361 (2016).

50. Williamson, M. P. Many residues in cytochrome c populate alternative states under equilibrium conditions. Proteins 53, 731-739 (2003).

51. Lee, D., Hilty, C., Wider, G. \& Wuthrich, K. Effective rotational correlation times of proteins from NMR relaxation interference. J. Magn. Reson. 178, 72-76 (2006)

52. Ryu, S., Liu, B., Yao, J., Fu, Q. \& Qin, F. Uncoupling proton activation of vanilloid receptor TRPV1. J. Neurosci. 27, 12797-12807 (2007).

53. Boukalova, S., Marsakova, L., Teisinger, J. \& Vlachova, V. Conserved residues within the putative S4-S5 region serve distinct functions among thermosensitive vanilloid transient receptor potential (TRPV) channels. $J$. Biol. Chem. 285, 41455-41462 (2010).

54. Aneiros, E. et al. The biophysical and molecular basis of TRPV1 proton gating. EMBO J. 30, 994-1002 (2011).

55. Jordt, S. E., Tominaga, M. \& Julius, D. Acid potentiation of the capsaicin receptor determined by a key extracellular site. Proc. Natl Acad. Sci. U. S. A. 97, 8134-8139 (2000).

56. McIntyre, P. et al. Pharmacological differences between the human and rat vanilloid receptor 1 (VR1). Br. J. Pharmacol. 132, 1084-1094 (2001).

57. Vandewauw, I. et al. A TRP channel trio mediates acute noxious heat sensing. Nature 555, 662-666 (2018).

58. Laursen, W. J., Schneidera, E. R., Merrimand, D. K., Bagriantseva, S. N. \& Gracheva, E. O. Low-cost functional plasticity of TRPV1 supports heat tolerance in squirrels and camels. Proc. Natl Acad. Sci. U. S. A. 113, 11342-11347 (2016).

59. Rath, P., Hilton, J. K., Sisco, N. J. \& Van Horn, W. D. Implications of Human Transient Receptor Potential Melastatin 8 (TRPM8) Channel Gating from
Menthol Binding Studies of the Sensing Domain. Biochemistry 55, 114-124 (2016).

60. Paramonov, A. S. et al. NMR investigation of the isolated second voltagesensing domain of human Nav1.4 channel. Biochim. Biophys. Acta Biomembr. 1859, 493-506 (2017)

61. Butterwick, J. A. \& MacKinnon, R. Solution structure and phospholipid interactions of the isolated voltage-sensor domain from KvAP. J. Mol. Biol. 403, 591-606 (2010).

62. Peng, D. et al. Purification and structural study of the voltage-sensor domain of the human KCNQ1 potassium ion channel. Biochemistry 53, 2032-2042 (2014).

63. Ng, H. Q. et al. Purification and structural characterization of the voltagesensor domain of the hERG potassium channel. Protein Expr. Purif. 86, 98-104 (2012).

64. Takeshita, K. et al. X-ray crystal structure of voltage-gated proton channel. Nat. Struct. Mol. Biol. 21, 352-357 (2014).

65. Sosa-Pagan, J. O., Iversen, E. S. \& Grandl, J. TRPV1 temperature activation is specifically sensitive to strong decreases in amino acid hydrophobicity. Sci. Rep. 7, 549 (2017).

66. Yang, F. \& Zheng, J. High temperature sensitivity is intrinsic to voltage-gated potassium channels. eLife 3, e03255 (2014).

67. Hazan, A., Kumar, R., Matzner, H. \& Priel, A. The pain receptor TRPV1 displays agonist-dependent activation stoichiometry. Sci. Rep. 5, 12278 (2015).

68. Singh, A. K., McGoldrick, L. L. \& Sobolevsky, A. I. Structure and gating mechanism of the transient receptor potential channel TRPV3. Nat. Struct. Mol. Biol. 25, 805-813 (2018)

69. Hughes, T. E. T. et al. Structural basis of TRPV5 channel inhibition by econazole revealed by cryo-EM. Nat. Struct. Mol. Biol. 25, 53-60 (2018).

70. Singh, A. K., McGoldrick, L. L., Twomey, E. C. \& Sobolevsky, A. I. Mechanism of calmodulin inactivation of the calcium-selective TRP channel TRPV6. Sci. Adv. 4, eaau6088 (2018).

71. Singh, A. K., Saotome, K., McGoldrick, L. L. \& Sobolevsky, A. I. Structural bases of TRP channel TRPV6 allosteric modulation by 2-APB. Nat. Commun. 9, 2465 (2018).

72. Huynh, K. W. et al. Structure of the full-length TRPV2 channel by cryo-EM. Nat. Commun. 7, 11130 (2016)

73. Wen, H. \& Zheng, W. Decrypting the heat activation mechanism of TRPV1 channel by molecular dynamics simulation. Biophys. J. 114, 40-52 (2018).

74. Boukalova, S., Teisinger, J. \& Vlachova, V. Protons stabilize the closed conformation of gain-of-function mutants of the TRPV1 channel. Biochim. Biophys. Acta 1833, 520-528 (2013).

75. Lomize, M. A., Pogozheva, I. D., Joo, H., Mosberg, H. I. \& Lomize, A. L. OPM database and PPM web server: resources for positioning of proteins in membranes. Nucleic Acids Res. 40, D370-D376 (2012).

76. Zhang, F., Swartz, K. J. \& Jara-Oseguera, A. Conserved allosteric pathways for activation of TRPV 3 revealed through engineering vanilloid-sensitivity. eLife 8, e42756 (2019).

77. Yin, Y. et al. Structural basis of cooling agent and lipid sensing by the coldactivated TRPM8 channel. Science 363, eaav9334 (2019).

78. Singh, A. K. et al. Structural basis of temperature sensation by the TRP channel TRPV3. Nat. Struct. Mol. Biol. 29, 994-998 (2019).

79. Vriens, J., Owsianik, G., Janssens, A., Voets, T. \& Nilius, B. Determinants of 4 alpha-phorbol sensitivity in transmembrane domains 3 and 4 of the cation channel TRPV4. J. Biol. Chem. 282, 12796-12803 (2007).

80. Ammann, C., Meier, P. \& Merbach, A. A simple multinuclear NMR thermometer. J. Magn. Reson. (1969) 46, 319-321 (1982).

81. Vranken, W. F. et al. The CCPN data model for NMR spectroscopy: development of a software pipeline. Proteins 59, 687-696 (2005).

82. Bond, C. S. \& Schuttelkopf, A. W. ALINE: a WYSIWYG protein-sequence alignment editor for publication-quality alignments. Acta Crystallogr. D: Biol. Crystallogr. 65, 510-512 (2009)

83. Delaglio, F. et al. NMRPipe: a multidimensional spectral processing system based on UNIX pipes. J. Biomol. NMR 6, 277-293 (1995).

84. Williamson, M. P. Using chemical shift perturbation to characterise ligand binding. Prog. Nucl. Magn. Reson. Spectrosc. 73, 1-16 (2013).

85. Gautier, A., Mott, H. R., Bostock, M. J., Kirkpatrick, J. P. \& Nietlispach, D. Structure determination of the seven-helix transmembrane receptor sensory rhodopsin II by solution NMR spectroscopy. Nat. Struct. Mol. Biol. 17, 768-774 (2010).

86. Wagner, G., Pardi, A. \& Wuethrich, K. Hydrogen bond length and proton NMR chemical shifts in proteins. J. Am. Chem. Soc. 105, 5948-5949 (1983).

87. Frauenfelder, H. et al. Thermal expansion of a protein. Biochemistry $\mathbf{2 6}$ 254-261 (1987).

88. Trainor, K., Palumbo, J. A., MacKenzie, D. W. S. \& Meiering, E. M. Temperature dependence of NMR chemical shifts: tracking and statistical analysis. Protein Sci. 29, 306-314 (2020). 
89. Iwahara, J., Tang, C. \& Marius Clore, G. Practical aspects of (1)H transverse paramagnetic relaxation enhancement measurements on macromolecules. $J$. Magn. Reson. 184, 185-195 (2007).

90. Van Horn, W. D., Beel, A. J., Kang, C. \& Sanders, C. R. The impact of window functions on NMR-based paramagnetic relaxation enhancement measurements in membrane proteins. Biochim. Biophys. Acta 1798, 140-149 (2010).

91. Veglia, G., Carolina Zeri, A., Ma, C. \& Opella, S. J. Deuterium/hydrogen exchange factors measured by solution nuclear magnetic resonance spectroscopy as indicators of the structure and topology of membrane proteins. Biophys. J. 82, 2176-2183 (2002).

92. Fitzkee, N. C. \& Bax, A. Facile measurement of (1)H-(1) $5 \mathrm{~N}$ residual dipolar couplings in larger perdeuterated proteins. J. Biomol. NMR 48, 65-70 (2010).

93. Mascioni, A. \& Veglia, G. Theoretical analysis of residual dipolar coupling patterns in regular secondary structures of proteins. J. Am. Chem. Soc. 125 , 12520-12526 (2003).

94. Mesleh, M. F., Veglia, G., DeSilva, T. M., Marassi, F. M. \& Opella, S. J. Dipolar waves as NMR maps of protein structure. J. Am. Chem. Soc. 124, 4206-4207 (2002).

95. Zweckstetter, M. NMR: prediction of molecular alignment from structure using the PALES software. Nat. Protoc. 3, 679-690 (2008).

96. Word, J. M., Lovell, S. C., Richardson, J. S. \& Richardson, D. C. Asparagine and glutamine: using hydrogen atom contacts in the choice of side-chain amide orientation. J. Mol. Biol. 285, 1735-1747 (1999).

97. Royer, C. A., Mann, C. J. \& Matthews, C. R. Resolution of the fluorescence equilibrium unfolding profile of trp aporepressor using single tryptophan mutants. Protein Sci. 2, 1844-1852 (1993).

\section{Acknowledgements}

The authors thank Charles R. Sanders for the KCNQ1-VSD plasmid. This study was supported by the National Institutes of Health R01GM112077 (W.D.V.H.) and the National Science Foundation \#1919716 (M.L. and W.D.V.H.)

\section{Author contributions}

W.D.V.H. designed the study. M.K., N.J.S., J.K.H., C.M.M., M.A.C., M.L., and W.D.V.H. conducted experiments. M.K., N.J.S., J.K.H., M.L., and W.D.V.H. analyzed the data.
M.K., N.J.S., M.L., and W.D.V.H. wrote the manuscript. B.R.C. wrote pulse programs for NMR measurements. All authors read and approved the final manuscript.

\section{Competing interests}

The authors declare no competing interests.

\section{Additional information}

Supplementary information is available for this paper at https://doi.org/10.1038/s41467020-18026-2.

Correspondence and requests for materials should be addressed to W.D.V.H.

Peer review information Nature Communications thanks Thomas Voets and other, anonymous, reviewers for their contributions to the peer review of this work. Peer review reports are available.

Reprints and permission information is available at http://www.nature.com/reprints

Publisher's note Springer Nature remains neutral with regard to jurisdictional claims in published maps and institutional affiliations.

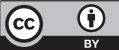

Open Access This article is licensed under a Creative Commons Attribution 4.0 International License, which permits use, sharing, adaptation, distribution and reproduction in any medium or format, as long as you give appropriate credit to the original author(s) and the source, provide a link to the Creative Commons license, and indicate if changes were made. The images or other third party material in this article are included in the article's Creative Commons license, unless indicated otherwise in a credit line to the material. If material is not included in the article's Creative Commons license and your intended use is not permitted by statutory regulation or exceeds the permitted use, you will need to obtain permission directly from the copyright holder. To view a copy of this license, visit http://creativecommons.org/ licenses/by/4.0/.

(C) The Author(s) 2020 